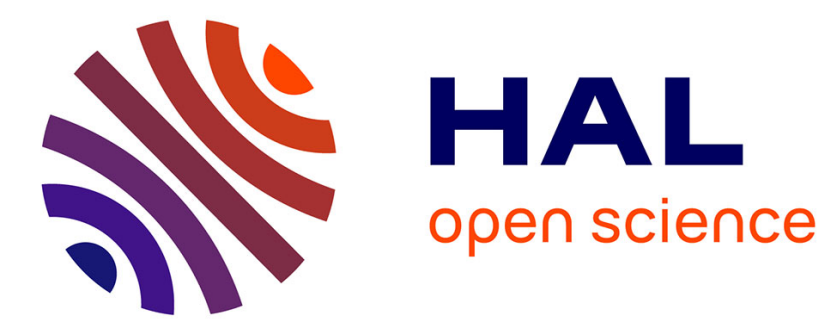

\title{
Wallis on Indivisibles
}

Antoni Malet, Marco Panza

\section{To cite this version:}

Antoni Malet, Marco Panza. Wallis on Indivisibles. Vincent Jullien. Seventeenth-Century Indivisibles Revised, Birkhäuser-Springer, pp.307-346., 2015, 10.1007/978-3-319-00131-9_14 . halshs-01172641

\section{HAL Id: halshs-01172641 https://shs.hal.science/halshs-01172641}

Submitted on 7 Jul 2015

HAL is a multi-disciplinary open access archive for the deposit and dissemination of scientific research documents, whether they are published or not. The documents may come from teaching and research institutions in France or abroad, or from public or private research centers.
L'archive ouverte pluridisciplinaire HAL, est destinée au dépôt et à la diffusion de documents scientifiques de niveau recherche, publiés ou non, émanant des établissements d'enseignement et de recherche français ou étrangers, des laboratoires publics ou privés. 


\title{
Wallis on Indivisibles
}

\author{
Antoni Malet* and Marco Panza** \\ *Univ. Pompeu Fabra (Barcelona) and MPIWG (Berlin) \\ **CNRS, IHPST (UMR 8590 of CNRS, University of Paris 1, and ENS Paris)
}

July 7, 2015

The present chapter is devoted, first, to discuss in detail the structure and results of Wallis's major and most influential mathematical work, the Arithmetica Infinitorum ([51]). Next we will revise Wallis's views on indivisibles as articulated in his answer to Hobbes's criticism in the early 1670s. Finally, we will turn to his discussion of the proper way to understand the angle of contingence in the first half of the 1680s. As we shall see, there are marked differences in the status that indivisibles seem to enjoy in Wallis's thought along his mathematical career. These differences correlate with the changing context of 17th-century mathematics from the 1650s through the 1680s, but also respond to the different uses Wallis gave to indivisibles in different kinds of texts - purely mathematical, openly polemical, or devoted to philosophical discussion of foundational matters.

\section{The Program of the Arithmetica Infinitorum}

Along his whole mathematical career, Wallis always argued for the foundational primacy of arithmetic over geometry. As we understand it, his main purpose was extending arithmetic so as to make it able to express numerically the relevant features of any sort of quantities. Hence, by advocating the foundational primacy of arithmetic over geometry, he was not arguing that geometry be dismissed or reduced to arithmetic, but rather that arithmetic should be so shaped as to make it possible to use its language in order to speak of geometric magnitudes and of all their quantitative properties and relations ${ }^{1}$. This point of view largely explains Wallis's use of indivisibles.

Indivisibles play a crucial role in Wallis's major mathematical work, the Arithmetica infinitorum, as well as in his De Sectionibus Conicis. Though both treatises first appeared together in the second volume of Wallis's Operum Mathematicorum, published in 1656 in Oxford ([53]) — where he was Savilian professor of geometry since 1649-, the De Sectionibus Conicis was composed slightly before the Arithmetica infinitorum ${ }^{2}$. Broadly speaking, one could say that the latter

\footnotetext{
${ }^{1}$ For a recent quite complete account of Wallis's mathematical production, cf. [23]. Another, more classical (but by far less complete) account is provided by [44]. According the former, Wallis's foundational "project" aimed at establishing a new branch of mathematics that Maierù suggests to term 'arithmet-algebra'. Our interpretation is quite different (cf. [35]): in our view, algebra was not understood by Wallis as a separate branch or discipline, but rather as a powerful formalism to be used in arithmetic, and, through it, also in geometry.

${ }^{2}$ For some details on the composition and the publication of Wallis's treatises, cf. [29], vol. I, 100, continuation
} 
treatise generalises some basic ideas already presented in the former within the restricted setting of a theory of conic sections. These ideas are based on an arithmetical understanding of indivisibles. In generalising them, Wallis firstly gets the quadrature and cubature of a quite large class of curves and solids of revolution - together with a wrong rectification of spirals-, then tackles, from a quite new perspective, the circle-squaring problem. The result is well-known ${ }^{3}$ : Wallis provides a mathematical argument supporting the conjecture that the ratio between a circle and the square constructed on its diameter cannot be expressed through a rational or surd number, and suggests an alternative way to get "the quadrature of the circle as far as the nature of numbers allows" ${ }^{4}$.

Wallis's basic ideas were close to those advanced by a number of contemporary mathematicians, like Fermat, Roberval, Pascal, and Mengoli $^{5}$, though the way these mathematicians applied them differ in many details from Wallis's, and there is no evidence he knew their methods and results at the time he published the De Sectionibus Conicis and the Arithmetica infinitorum. In the dedicatory letter of the latter ([51], [iv-vi], and [58], 1-3), he claims that his own method "takes its beginning where Cavalieri's method of Indivisibles ends", was inspired by Cavalieri's method as presented in Torricelli's writings ${ }^{6}$, and aims at using it for tackling the circle-squaring problem. Still, the way he continues makes evident a significant shift:

The ratio of a cone [composed] of an infinite number of circles to a cylinder [composed] of so many [circles] was already known, namely 1 to 3 ; moreover all the diameters in a triangle along the axis of a cone, to so many [diameters] in a parallelogram along the axis of the cylinder, are (as is known) as 1 to 2. Equally all the circles in a parabolic conoid, to so many circles in a cylinder were known to have the ratio of 1 to 2; moreover all the diameters of the former to the diameters of the latter are as 2 to 3 . It was also clear that the straight lines of a triangle are arithmetically proportional, or as $1,2,3,3$, etc. and so the circles of a cone ([which are] in a duplicate ratio than the diameters) [are] as 1, 4, 9, etc. In the same way the circles of a parabolic conoid ([which are] in a duplicate ratio than the ordinates, that is, in the ratio of the diameters) [are] as $1,2,3$, etc. and therefore their diameters as $\sqrt{1}, \sqrt{2}, \sqrt{3}$, etc. indeed in a sub-duplicate ratio than their circles. I hoped it might therefore be possible that, from the known ratios of other series of circles, or squares (which comes down to the same thing), to as many equals, there might be found also the ratio of their diameters or sides to as many equals. Moreover if I could find this

of footnote 23; [45], 148a; [47]; [48], 165-167; [58], xvii-xxi). Likely, the De Sectionibus Conicis was already completed in 1652, while the Arithmetica infinitorum was begun during the same year, but concluded later. Both of them were firstly printed in 1655 , but only few copies were possibly distributed, while others were stored and then inserted in the second volume of the Operum Mathematicorum, with separate pagination and title pages, respectively dated 1655 (for the De Sectionibus Conicis: [50]) and 1656 (for the Arithmetica infinitorum: $[51])$.

${ }^{3}$ On Wallis' method, and, more generally, on the Arithmetica infinitorum, cf.: [6], II, 899-904; [30]; [40], 387-391; [44], 15-64; [59], 236-243 and 319-321; [2], 208-211; [14], 87-95; [32], 163-211; [24], 35-41; [47], 1-8; [48], 155-165; [58], xi-xxxiii; [33], 49-81; [23], 117-197; [13]; [17], 139-144.

${ }^{4}$ Cf. [51], [xiii], and [58], 7. Our quotes from the Arithmetica infinitorum come, with few minor local changes, from the recent English translation of offered by J. A. Stedall: [58]. We also refer however to the original edition.

${ }^{5}$ Cf. [59], 319; [2], 150-156; [24], 36; [26], 259 et 265-266; [27], 108-109; [58], xiv.

${ }^{6}$ Wallis explicitly says that he did never read Cavalieri's treatises: "Cavalieri himself I did not have to hand, and I sought it in vain at various book-sellers" ([51], [iv], and [58], 2). According Stedall ([58]., xv), Cavalieri's books, were, indeed "almost impossible to obtain ", so that Wallis "learned of his work at second hand from the more easily available Opera of Torricelli". 
by some general method, the quadrature of the circle would be sufficiently in sight. For since, as was already known, all the parallel circles in a sphere, to as many in a cylinder, are as 2 to 3 , if thence there could be known the ratio of all the diameters of the former to the diameters of the latter, there would be found what was sought: for certainly the diameters of the former constitute a circle, the latter the square of the diameter. Thus a geometric problem is reduced to a purely arithmetic one. follows.

By using a convenient notation (which is not Wallis's), this argument can be rephrased as

Let $\mathfrak{X}, \mathfrak{Y}$, and $\mathfrak{Z}$ be, respectively, a cone, a parabolic conoid, and a cylinder with the same altitude and the same base. Conceive each of these solids as being composed by the same infinite number of circles. Let these circles be $\mathcal{X}_{i}, \mathcal{Y}_{i}, \mathcal{Z}_{i}$, respectively. Circles $\mathcal{X}_{i}$ and $\mathcal{Y}_{i}$ would be all different to each other, whereas circles $\mathcal{Z}_{i}$ would be all equal to each other; let us say that they would be all equal to the common base $\mathcal{Z}$ of $\mathfrak{X}, \mathfrak{Y}$, and $\mathfrak{Z}$, so that $\mathcal{Z}_{i}=\mathcal{Z}$, for any $i$. As $\mathfrak{X}: \mathfrak{Z}=1: 3$, and $\mathfrak{Y}: \mathfrak{Z}=1: 2$, it would follow that

$$
\sum \mathcal{X}_{i}: \sum \mathcal{Z}_{i}=1: 3 \quad ; \quad \sum \mathcal{Y}_{i}: \sum \mathcal{Z}_{i}=1: 2 .
$$

where ' $\sum \mathcal{X}_{i}^{\prime}$ ', ' $\sum \mathcal{Y}_{i}$ ' and ' $\sum \mathcal{Z}_{i}$ ' denote the totalities of circles composing $\mathfrak{X}, \mathfrak{Y}$, and $\mathfrak{Z}$, respectively. Let now $x_{i}, y_{i}, z_{i}$ be, respectively the diameters of the circles composing these solids. Once more, diameters $x_{i}$ and $y_{i}$ would be all different to each other, whereas diameters $z_{i}$ would be all equal to each other; let us say that they would be all equal to the diameter $z$ of $\mathcal{Z}$, so that $z_{i}=z$, for any $i$. Taken together, the diameters $x_{i}, y_{i}, z_{i}$ would respectively compose a triangle, a surface delimited by a parabola and a rectangle. As these figures would have the same base and the same altitude, it would follow that

$$
\sum x_{i}: \sum z_{i}=1: 2 \quad ; \quad \sum y_{i}: \sum z_{i}=2: 3
$$

where the symbol ' $\sum$ ' is used as before.

Now, both the circles $\mathcal{Y}_{i}$ and the diameters $x_{i}$ would increase in arithmetic proportion, $i$. $e$. as the terms of the sequence $1,2,3, \ldots$, whereas the circles $\mathcal{X}_{i}$ would increase as the terms of the sequence $1,4,9, \ldots$ and the diameters $y_{i}$ as the terms of the sequence $\sqrt{1}, \sqrt{2}, \sqrt{3}, \ldots$ On the other hand, as the circles $\mathcal{Z}_{i}$ and the diameters $z_{i}$ would be all respectively equal to each other, they would increase as the terms of the sequence $h, h, h, \ldots$, where $h$ is any number whatsoever (we may assume it to be a positive integer). Hence, as the greatest of the circles $\mathcal{X}_{i}$ and $\mathcal{Y}_{i}$ would be equal to every one of the $\mathcal{Z}_{i}$, and the greatest of the diameters $x_{i}$ and $y_{i}$ would be equal to every $z_{i}$, there should be purely arithmetic results capable of expressing the relevant content of proportions (1) and (2). If these results were found, these last proportions could be drawn from it.

Suppose now that the common altitude of $\mathfrak{X}, \mathfrak{Y}$, and $\mathfrak{Z}$ be equal to the diameter $z$ of their common base $\mathcal{Z}$, and that $\mathfrak{W}$ be a sphere with this same diameter. Conceive this sphere as being composed by as many different circles $\mathcal{W}_{i}$ (the greatest of which is of course equal to $\mathcal{Z}$ ) as compose $\mathfrak{X}, \mathfrak{Y}$, and $\mathfrak{Z}$. Since $\mathfrak{W}: \mathfrak{Z}=2: 3$, it would follow that

$$
\sum \mathcal{W}_{i}: \sum \mathcal{Z}_{i}=2: 3=\sum y_{i}: \sum z_{i}
$$

Finally, let $w_{i}$ be the diameters of circles $\mathcal{W}_{i}$, respectively. Taken together, they would compose a circle of diameter $z$, whereas the same number of diameters all equal to $z$ would compose a 
square of side $z$. Hence, the ratio of a circle and the square on its diameter would the same as the ratio of $\sum w_{i}$ and $\sum z_{i}$.

Wallis's point is that it should be possible to reduce the geometric problem of squaring the circle to the arithmetical problem of finding this last ratio by relying on purely arithmetic results capable of expressing the relevant content of proportions (1) and (2). This suggests a twofold program: to look for a way for stating and founding appropriate results about the ratio of arithmetic series; to show that these results provide the solution of a number of geometric problems, the most important of which is the quadrature of the circle. Indivisibles enter this program as tools to reach the latter task: they are used to connect geometry to arithmetic.

\section{Wallis's Basic Idea}

Apart from the foregoing explicit mention in the dedicatory letter, indivisibles enter into the Arithmetica infinitorum only implicitly or indirectly. The first indirect mention is made in proposition III. To understand it, let us consider first propositions I and II.

The former is a problem, and requires to find the ratio between "a series of quantities in arithmetic proportion [...] continually increasing, beginning from a point or $0[\ldots]$, thus as $0,1,2,3,4$,etc." and "the sum of as many terms equal to the greatest" ([51], 1, and [58], 13). As Stedall remarks ([58], 13, footnote 3), by taking his "series" to begin "from a point or 0", Wallis is here implying that its terms are either geometric magnitudes or numbers. Still, to solve the problem, he takes nothing but integer non-negative numbers into account. This shows that he conceives them as capable of expressing the relevant relations of any sequence of quantities increasing in arithmetic proportion. This being admitted, and supposing that the relevant series only include a finite number of terms, Wallis solves the problem by appealing to incomplete induction $^{7}$, which allows him to conclude that

$$
\frac{\sum_{i=0}^{h} i}{h(h+1)}=\frac{1}{2},
$$

for any positive integer (finite) number $h^{8}$.

Proposition II, a theorem, states that the same result holds when the series includes an infinite number of terms. No real proof is offered. Wallis first claims that "there will be no reason to distinguish" the two cases. Next explains that, if the first term of the series is 0 , the second 1, the last $l$, and the series includes $m$ terms, then the sum is $\frac{l+1}{2} l$ or $\frac{1}{2} m l$. If we admit that ' 1 ' is a numeral, then also ' $l$ ' should be a generic numeral and refer to the same number as ' $h$ ' in (4). One would then have $l=h=m-1$, and $\frac{l+1}{2} l=\frac{1}{2} m l=\frac{h(h+1)}{2}$. Wallis point seems then that the equality $\frac{\frac{h(h+1)}{2}}{h(h+1)}=\frac{1}{2}$ holds both if $h$ is a finite or an infinite positive integer. The most relevant point is another, however. As Wallis himself remarks, $\frac{1}{2} m l$ is the sum of the relevant series whatever its second term might be. In other terms, $\frac{1}{2} m l$ is the sum of any arithmetic progression "continually increasing, beginning from a point or 0 " including $m$ terms

\footnotetext{
${ }^{7}$ Incomplete induction is largely used in the Arithmetica infinitorum: many results are proved for the first integer non-negative numbers, then assumed to hold for all such numbers. Coeval and posterior commentators have largely discussed this issue (cf., for instance, [24], 36-38 and [58], xxiv-xxv).

${ }^{8}$ Of course, Wallis does not use the $\sum$-notation for series. We use it for short.
} 
and whose last term is $l$. This result is then perfectly independent of the nature of $l$. If the terms of the series are taken to be whatsoever sort of quantities, the first of which is null, and its reason is taken to be $e$, Wallis's proposition is thus equivalent to the following equalities:

$$
\sum_{i=0}^{h} i e=\frac{1}{2}(h+1) h e \quad \text { and } \quad \frac{\sum_{i=0}^{h} i e}{(h+1) h e}=\frac{1}{2}
$$

where $h$ is either a finite or an infinite integer positive number, and $e$ a quantity of whatsoever sort.

Proposition III is a corollary of proposition II ([51], 2, and [58], 14-15):

Therefore, a triangle to a parallelogram (on an equal base and of equal altitude) is as 1 to 2 .

And this is the proof.

For the triangle consists, as it were, of an infinite number of parallel straight lines in arithmetic proportion, starting from a point, of which the greatest is the base (as we showed in Propositions I and II of our book De sectionibus conicis); and the parallelogram consists of as many [straight lines] equal to the base (as is clear).

The indirect mention to the method of indivisibles comes with the reference to propositions I-II of the De sectionibus conicis.

Already, in the dedicatory letter of this treatise, Wallis is quite explicit in endorsing this method $([50],[\mathrm{vi}])^{9}$ :

[...] you will see me, from the very beginning, both in this treatise and in the following one (which is the twin of this) ${ }^{10}$, to lay under Cavalieri's method of indivisibles which is now far and wide accepted by geometers.

Proposition I makes then perfectly clear what this means $([50], 4)^{11}$ :

I suppose, from the beginning (after Bonaventura Cavalieri's Geometriam Indivisibilium) that any [portion of] plane consists, as it were, of infinitely many parallel [straight] lines, or rather (as I would prefer) of infinitely many parallelograms equally high, the altitude of each of which is $\frac{1}{\infty}$ of the total altitude, that is, an infinitely small aliquot part (for ' $\infty$ ' denotes an infinite number), so that the altitude of all [such parts] taken together is equal to the altitude of the figure.

Wallis seems then to reduce Cavalieri's method to the supposition that any geometric extension is composed by a infinite number of elements, which either have a smaller dimension, or are homogenous with this extension but have an infinitely small size in the appropriate dimension. He not only avoids to make a choice among these possibilities, but he also seems to consider the entire discussion opposing them as quite immaterial ([50], 4-5):

\footnotetext{
${ }^{9}$ Unless explicitly indicated, translations from the De sectionibus conicis are ours.

${ }^{10}$ This is the Arithmetica infinitorum, of course.

${ }^{11}$ The following translation is based on that offered by Stedall: [58], xxii.
} 
Each way of explaining things (either by infinitely many parallel [straight] lines, or by infinitely many equally high parallelograms placed between these infinitely many lines) will result in the same thing. Indeed, a parallelogram whose altitude is supposed to be infinitely small, that is, null (for an infinitely small quantity is just a no quantity), hardly is something else than a line. (In this at least they differ, that this line is assumed to be dilatable, or to have at least a little bit of thickness, such that, by infinite multiplication, it might finally acquire a certain altitude or latitude, namely so much as is the altitude of the figure.) Therefore in what follows (partly because it seems proper to preserve the way of speaking in Cavalieri's method of indivisibles, and partly also for brevity's sake), sometimes we shall call with the name of lines, rather than [with that] of parallelograms, those parts of figures which are infinitely small (or have infinitely small altitude) - at all events when no determinate altitude [of these parts] is taken into consideration. But when a determinate altitude will be taken into consideration (as sometimes it will), [some] ratio of that small altitude is always to be had, so that, if infinitely multiplied, it may be assumed to equal the whole altitude of the figure.

Finally, proposition II introduces no correction, and merely applies this understanding to triangles.

This leaves open two possibilities for understanding the proof of proposition III of the Arithmetica infinitorum. Let a triangle and a parallelogram, both of altitude $k$ and base $l$, be given. Under the homogeneity option, they are supposed to be composed by the same infinite number of parallelograms, whose common altitude is $\frac{1}{\infty} k$. Clearly, these parallelograms stay to each other as their bases. Hence only these bases are relevant for the purpose of the proof. Insofar as, under the inhomogeneity option, the elements of the two given figures are just these bases, the two options are operationally equivalent (that is, they are so if the continuum-composition issue is left apart). The elements composing the given parallelogram (under the latter option), or the bases of these elements (under the former option) are all equal to $l$, whereas the elements composing the given triangle or the bases of these elements are to each other as $\frac{0}{\infty} k, \frac{1}{\infty} k$, $\frac{2}{\infty} k, \ldots, \frac{\infty}{\infty} k$. Hence, for any integer non-negative number $i$, the $i$-th element of the triangle or its base are to $\frac{i}{\infty} k$ as $l$ is to $k$, and are thus equal to $\frac{i}{\infty} l^{12}$. Supposing that $h=\infty$ and $e=\frac{1}{\infty} l=\frac{l}{h}$, one gets then from (4) or (5), that the given triangle is to the given parallelogram as 1 to 2 , namely:

$$
\left[\frac{\sum_{i=0}^{h} i \frac{l}{h}}{h(h+1) \frac{l}{h}}\right]_{h=\infty}=\left[\frac{\sum_{i=0}^{h} i}{h(h+1)}\right]_{h=\infty}=\frac{1}{2} .
$$

One can then distinguish three stages in Wallis' argument. The first is purely arithmetic; it consists in calculating the value of $\frac{\sum_{i=0}^{h} i}{h(h+1)}$ for whatever integer positive number $h$ either finite or infinite. The second is purely geometric; it consists in equating the ratio of a triangle and a parallelogram having the same altitude and the same base to the ratio of two aggregates of

\footnotetext{
${ }^{12}$ Remember that in Wallis's notation ' $\infty$ ' denotes an integer number, though infinite, so that $\frac{0}{\infty}=0$ and $\frac{\infty}{\infty}=1$. This is made clear in Wallis's proof of proposition III of the De sections conicis, on which we shall come back below: cf. [50], 8-9, and [23], 61-62.
} 
appropriate components. The third consists in connecting the two first stages, so as to assign to this last ratio the same value as that of $\left[\frac{\sum_{i=0}^{h} i}{h(h+1)}\right]_{h=\infty}$. Because of the operational equivalence of the homogeneity and the inhomogeneity options, both the first and the third stages are independent of the argument that the second is based on: whatever this argument might be, it leads to the conclusion that the ratio of the two given figures is equal to $\left[\frac{\sum_{i=0}^{h} i \frac{l}{h}}{h(h+1) \frac{l}{h}}\right]_{h=\infty}$, which is in turn obviously equal to $\left[\frac{\sum_{i=0}^{h} i}{h(h+1)}\right]_{h=\infty}$. Notice that Wallis's argument does not hinge, then, on the nature of indivisibles, but rather on the rate of change of the chords of the triangle which, independently of how indivisibles are understood, is reflected by the relation that the terms of the arithmetic sequence $0,1,2, \ldots$ have to each other.

Things do not go this way, however, in proposition III of the De sectionibus conicis. This is a theorem stating that "the area of a triangle is equal to the base multiplied by half the altitude" ([50], 8). Rather than determining a ratio between two given polygons, as in proposition III of the Arithmetica infinitorum, Wallis determines here the value of one of them, namely of the triangle's "area". Notice nevertheless that Wallis has defined neither the area of a polygon nor the product of two geometrical magnitudes. Still, he arises these difficult matters neither in De sectionibus conicis, nor in the Arithmetica infinitorum, although he rather vaguely dealt with these topics in chapters XXII and XXIV of the contemporary Mathesis universalis: [54], 192-202 and 209-221). This matter apart, most relevant for our purposes is the very proof Wallis provides. This is grounded on the "very well known rule", according to which the sum of an arithmetical progression is equal to the sum of its first and last terms multiplied by half the number of terms. Wallis remarks that if the first term is either the number 0 or a "a point in magnitude", then the sum of its first and last terms is equal to the last one. Therefore, if the terms of the progression are the elements of a triangle and their number is $\infty$, this aggregate is nothing but the base $B$ of such a triangle and therefore the sum of the progression is $\frac{\infty}{2} B$. Finally, he observes that the "thickness [crassities]" of each one of these elements ("lines or parallelograms") is $\frac{1}{\infty}$ of the altitude $A$ of the triangle, so that its area is just $\frac{1}{\infty} A \frac{\infty}{2} B=\frac{1}{2} A B$.

Clearly, this is not only an infinitary argument, but it rests on the assumption that the relevant elements (that is, the invisibles composing the triangle) have a thickness (and then two dimensions). It is still significant that Wallis assigns thickness to "lines or parallelograms", and avoids explicitly endorsing the homogeneity option. This confirms that he is not interested in the metaphysical foundations of the method of indivisibles.

Now, why does he indulge, then, in so elementary a question as the "area" of a triangle or its ratio with a rectangle? The answer differs according whether the De sectionibus conicis or the Arithmetica infinitorum are taken into account. Proposition III of the former treatise provides a model for defining cones as aggregates of an infinity of parallel circles or very thin cylinders (proposition V: [50], 10-14) 13 $^{13}$ so as to get an understanding of conic sections as being

\footnotetext{
${ }^{13}$ Here is the statement of this proposition: "If the infinitely many parallel straight lines that a triangle is supposed to consist of were diameters of as many parallel circles, it would be formed a solid figure which is called 'cone'." Proposition VI (ibid., 14-15) offers an analogous definition also for pyramids.
} 
ipso facto endowed with their intrinsic ordinates orthogonal to the axes (proposition VII: [50], 15-22) ${ }^{14}$. Proposition III of the latter treatise provides, instead, a paradigm for a new method of quadrature and cubature. The next section is devoted to expound this method.

\section{Wallis's Method of Quadrature and Cubature}

The arguments Wallis relies on in propositions II and III of the De sectionibus conicis and in proposition III of the Arithmetica infinitorum apply both to rectilinear and to curvilinear triangles, provided that in the latter ones the chords parallel to the base vary as the terms of an arithmetical progression. This is made manifest by the figures appended to these propositions (figs. 2a and 2b: fig 2a is appended to proposition II of the De sectionibus conicis; fig 2b is appended to propositions III of the De sectionibus conicis and of the Arithmetica infinitorum). Still, this is not all what makes the first three propositions of the latter treatise relevant. What is much more important is that they suggest a method for proving many other results.

\footnotetext{
${ }^{14}$ In the following propositions of the first part of his treatise (propositions VIII-XX: [50], 22-45), Wallis relies then on this understanding in order to prove the basic properties of conics directly expressed through equations involving these ordinates and the relative abscises, and to show how other solids can be generated by conics. For example, in proposition XII ([50], 22), he remarks that the diameter SB (fig. 1) of each circle composing a cone is cut by the axis of a parabola generated from this cone in a point $\mathrm{D}$ such that both SD and the ratio of DA and DB are constant (since the axis AP is parallel to VS, to the effect that each triangle ADB is similar to each triangle VSB), and this is then also the case of the fourth proportional between DA, SD and DB. If one takes then this fourth proportional to be the segment $\mathrm{AL}$, and one observes that, because of the properties of circles, the orthogonal ordinate OD of the parabola is mean proportional between SD and DB (as observed in proposition VIII: [50], 27-32), it follows that this same ordinate is also mean proportional between DA and DB, to the effect that $\mathrm{DO}^{2}=\mathrm{AD} \times \mathrm{AL}, i . e . p^{2}=l d$, in Wallis's notation, or, $y^{2}=p x$, in our modern notation. Once these basic properties proved, in the second part of the treatise (propositions XXI-XLIV: [50], 46-103) Wallis relies on the equations expressing them for studying conics as such, or "absolutely [absolutè]", that is, without any consideration of the generator cones.
}

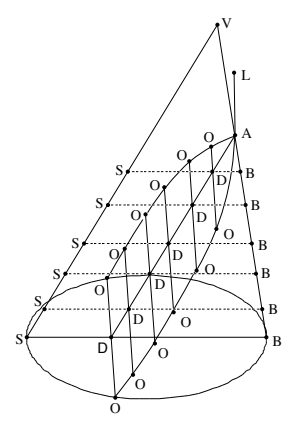

Fig. 1 

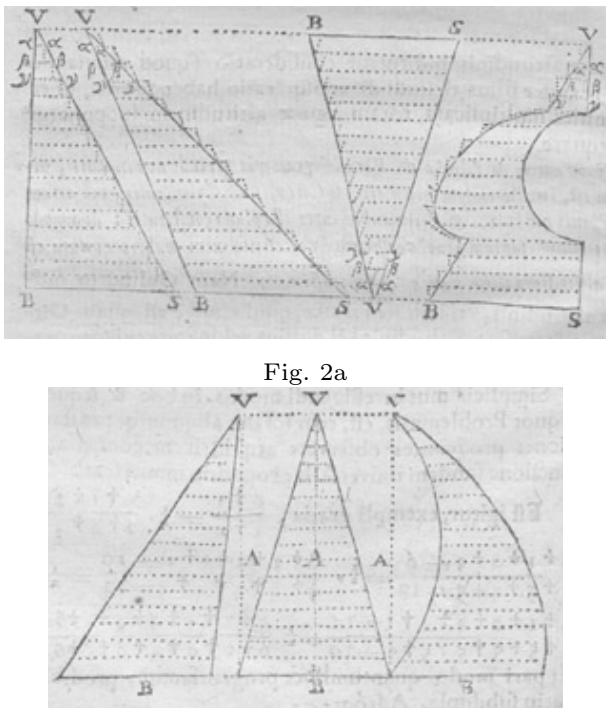

Fig. 2b

Some of them are direct corollaries of propositions I and II of this treatise. This is the case of those stated in proposition IV ([51], 3, and [58], 15-16): a parabolic pyramid and a parabolic conoid $^{15}$ are respectively to a prism and to a cylinder with the same altitude and the same base as 1 to 2. The reason is obvious: the parallel polygons and circles (or very thin prisms and cylinders) composing these solids are also respectively to each other as the terms of an arithmetic progression. Wallis thought possible an analogous argument would do for rectifying Archimedean spirals: taken together, propositions V-IX ([51], 3-7, and [58], 16-19) state that the arc of an Archimedean spiral going from the centre $M$ up to any point $T$ (fig. 3) is to the corresponding arc of circle of radius MT, plus the complete circumference of this circle taken as many times as complete revolutions have to be completed for going from $\mathrm{M}$ to $\mathrm{T}$ along the spiral itself, as 1 to 2 . This is wrong ${ }^{16}$. Wallis become probably aware of his error after the impression of these propositions and added a scholium to proposition XIII where he tries to save his statements by maintaining that the term 'spiral' should be understood in them as referring not to the Archimedean spiral properly, but rather to the "aggregate of all the arcs of similar sectors, infinite in number, of which consists that figure [which is] inscribed in the true spiral [and results] from the infinite number of sectors [of it]" ([51], 10 and [58], 21) ${ }^{17}$. The crucial

\footnotetext{
${ }^{15}$ These solids are defined by Wallis in an analogous way as cones in proposition IX of the De sectionibus conicis ([50], 23-24).

${ }^{16}$ The correct result had been proved by Roberval and made public by Mersenne in 1644 ([28], 129-131: Hydraulica, prop. XXV, cor. II)

${ }^{17}$ Our translation significantly differs from Stedall's, but is far from indisputable. The very Latin text is ambiguous: "Aggregatum omnium arcuum Sectorum similium, numero infinitorum, ex quibus constat figura illa ex infinitis numero Sectoribus Spirali vera inscripta" (to be precise, the adjective 'vera' is added only in the edition of the Opera Mathematica, where Wallis also introduced small changes in the statements and proofs of propositions V-IX so as to refer to the spiral as to a "spurious [spuria]" one): [57], vol. I, 370). Our translation is based on the understanding of 'ex infinitis numero Sectoribus' as indicating that from which the relevant figure results. We suggest, indeed, that Wallis was reasoning as follows: consider a sector MTT of an Archimedean spiral, and inscribe in it a curvilinear triangle resulting from this sector by replacing the arc of spiral TT with an arc of circle, then make the same for all the sectors of the spiral; the curve that the term 'spiral' should refer
} 
point of his wrong proof consists in arguing that, if the angles $\widehat{\mathrm{TMT}}$ are equal to each other and infinitely small, then the arcs delimitating the sectors TMT increase as the vector radii MT, and are thus in arithmetic proportion to each other. This would be correct, however, only if the arcs TT were replaced by arc of circles, which explains Wallis's tentative amendment ${ }^{18}$.

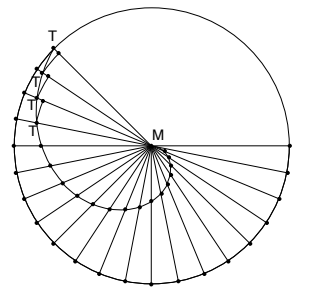

Fig. 3

This error was certainly brought about by Wallis's eagerness to draw as many geometric consequences as possible from the quite simple arithmetic results stated in the two first propositions of his treatise. But it is clear that more powerful arithmetic results are needed for getting more significant geometric outcomes through arguments analogous to the proof of proposition III.

The simplest of these outcomes concern the quadrature of parabolas of any integer positive order $n$. These are curves like AO (fig. 4) endowed with an axe AD and such that, if AT is the tangent at the vertex $A$, then for any pair of points $T$ and $T$ on this tangent the corresponding pair of segments TO and TO parallel to the axis $\mathrm{AD}$ are in $n$-plicate ratio of the corresponding pair of segments DO and DO parallel to AT (with $n$ whatever integer positive number) ${ }^{19}$.

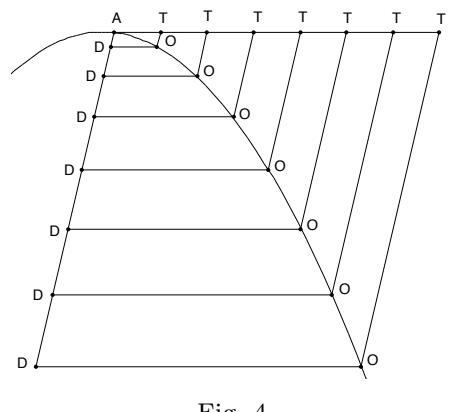

Fig. 4

If $n=1$, AO reduces to a straight line and the surfaces AOT reduces to rectilinear triangles. This is the case considered in proposition III. If $n=2$ or $n=3$, AO reduces, respectively, to an ordinary parabola or to a cubic parabola. The arithmetic results allowing to deal with these

to would then be that which is formed by all the arcs of circle got in this way.

${ }^{18} \mathrm{Cf}$. footnote (17), above. On the mathematical and historical details and implications of Wallis's error, cf. [29], III, 308-311 (footnote 704); [20], 117-125; [58], xxiii and 16, footnote 9. Other results connected with this wrong rectification of the Archimedean spirals are provided in propositions X-XVIII ([51], 7-15 and [58], 19-26).

${ }^{19}$ In the language of the theory of proportions, this means that for any pair of segments $\mathrm{AT}_{i}$ and $\mathrm{AT}_{j}$ (with $i$ and $j$ integer positive indexes) the corresponding pair of segments $\mathrm{T}_{i} \mathrm{O}_{i}=\mathrm{AD}_{i}$ and $\mathrm{T}_{j} \mathrm{O}_{j}=\mathrm{AD}_{j}$ are such that

$$
\mathrm{T}_{i} \mathrm{O}_{i}=\mathrm{T}_{j} \mathrm{O}_{j}=\alpha_{0}: \beta \quad \text { if and only if } \quad \mathrm{AT}_{i}: \mathrm{AT}_{j}=\alpha_{0}: \alpha_{1}=\alpha_{1}: \alpha_{2}=\ldots=\alpha_{n-1}: \beta
$$

where $\alpha_{k}(k=0, \ldots, n-1)$ and $\beta$ are appropriate quantities. 
cases are respectively stated in propositions XIX-XXI and XXXIX-XLI ([51], 15-17 and 31-32, and [58], 26-27 and 40). Wallis begins by observing (without proof) that for whatsoever finite integer positive number $h$, the following equalities hold

$$
\frac{\sum_{i=0}^{h} i^{2}}{h^{2}(h+1)}=\frac{1}{3}+\frac{1}{6 h} \quad \text { and } \quad \frac{\sum_{i=0}^{h} i^{3}}{h^{3}(h+1)}=\frac{1}{4}+\frac{1}{4 h} .
$$

Then, he argues that, "if one continues to infinity", the terms $\frac{1}{6 h}$ and $\frac{1}{4 h}$ "will vanish completely" ([51], 16 and 22, and [58], 27 and 40). Finally, he concludes that "an infinite continually increasing series of quantities [that are] in duplicate [resp. triplicate] ratio of arithmetic proportionals (or as a series of square [resp. cubic] numbers) beginning from a point or 0 is to a series of as many [terms] equal to the greatest as 1 to 3 [resp. 4]" ([51], 17, 32, and [58], 27, 40) ${ }^{20}$. Supposing that $e$ is a positive quantity of whatever sort, this corresponds to argue that:

$$
\left[\frac{\sum_{i=0}^{h} i^{2} e}{(h+1) h^{2} e}\right]_{h=\infty}=\left[\frac{\sum_{i=0}^{h} i^{2}}{h^{2}(h+1)}\right]_{h=\infty}=\frac{1}{3} \quad \text { and } \quad\left[\frac{\sum_{i=0}^{h} i^{3} e}{(h+1) h^{3} e}\right]_{h=\infty}=\left[\frac{\sum_{i=0}^{h} i^{3}}{h^{3}(h+1)}\right]_{h=\infty}=\frac{1}{4} \text {. }
$$

To apply these results to the quadrature of parabolas or cubic parabolas, suppose that AO is such a curve, and the differences TT are all equal to each other and to the first of segments AT, so that the segments TO are to each other in duplicate or triplicate ratio of arithmetic proportionals ${ }^{21}$. By reasoning like in the proof of proposition III, it follows that each surface AOT is to the corresponding parallelogram ADOT as 1 to 3 or as 1 to 4 , and consequently each surface ADO is to the same parallelogram as 2 to 3 or as 3 to 4 . This is just what Wallis claims, without any further explicit justification, in propositions XXIII and XLII, respectively ([51], 17-18, 33, and [58], 23, 40-41) ${ }^{22}$.

\footnotetext{
${ }^{20}$ Because of Wallis's repetitive style, it is difficult to say which parts of this arguments pertains to each proposition. In propositions XX and XL ([51], 16, 32, and [58], 27, 39-40), Wallis claims that the sums of the relevant series are respectively: $\frac{l+1}{3} l^{2}+\frac{1}{6 l} l^{2}$ or $\frac{m}{3} l^{2}+\frac{m}{6 m-6}$, and $\frac{l+1}{4} l^{3}+\frac{l+1}{4 l} l^{3}$ or $\frac{m}{4} l^{3}+\frac{m}{4 l} l^{3}$, where $l$ is the last and greatest term and $m$ the number of terms of these series. If these series are $\sum_{i=0}^{h} i^{2} e$ and $\sum_{i=0}^{h} i^{3} e$, respectively (where $e$ is a positive quantity of whatsoever sort), this is correct, however, only if $l$ is the rang (or index) of the last term and not this very term, that is, only if $l=h$ and $m=h+1$. It is thus hard to decide whether Wallis is only referring to the numerical series $\sum_{i=0}^{h} i^{2}$ and $\sum_{i=0}^{h} i^{3}$ or extends his results to series of whatsoever sorts of quantities. This ambiguity apart, Wallis's way of writing his sums suggests that he did possibly establish his results by relying on the equalities $\sum_{i=0}^{h} i^{2}=\frac{h(h+1)(2 h+1)}{6}$ and $\sum_{i=0}^{h} i^{3}=\frac{h^{2}(h+1)^{2}}{4}$, rather than by induction, as implicitly suggested, instead, by the proofs of propositions XIX and XXIX: cf. [13], 56-57.

${ }^{21}$ According to the condition stated in footnote (19), this means that if $\mathrm{AT}_{i}$ and $\mathrm{AT}_{j}$ (with $i$ and $j$ are integer positive indexes) are so taken that $\mathrm{AT}_{i}: \mathrm{AT}_{j}=i: j$ - to the effect that $\beta=\frac{j^{2}}{i}$ or $\beta=\frac{j^{3}}{i^{2}}$, then the corresponding segments $\mathrm{T}_{i} \mathrm{O}_{i}=\mathrm{AD}_{i}$ and $\mathrm{T}_{j} \mathrm{O}_{j}=\mathrm{AD}_{j}$ are such that $\mathrm{T}_{i} \mathrm{O}_{i}: \mathrm{T}_{j} \mathrm{O}_{j}=i^{2}: j^{2}$ or $\mathrm{T}_{i} \mathrm{O}_{i}: \mathrm{T}_{j} \mathrm{O}_{j}=i^{3}$ : $j^{3}$, respectively.

${ }^{22}$ By reasoning as in the case of triangles, using the same notation, and admitting multiplication on segments, this could have been justified as follows. Let $k$ and $l$ be respectively the greatest of segments AT and TO, that is, the two sides of a surface AOT that is to be squared. The differences TT and the first of segments AT are
} 
Proposition XXI can also provide a base for cubing cones and pyramids, and for squaring Archimedean spirals and getting other related outcomes. The former results are stated in proposition XXII ([51], 17, and [58], 28) ${ }^{23}$, the latter in propositions XXIV-XXXVIII ([51], 18-28, and $[58], 29-36)^{24}$.

Once these results obtained, Wallis does not indulge with looking for other analogous applications of the second equality (8), but rather goes ahead, in propositions XLIII-XLV ([51], 32-36, and [58], 41-43) to generalise his conclusions. He begins by extending equalities (8) to any increasing progression $\left\{i^{n} e\right\}_{i=0}^{h}(n=0,1,2, \ldots)$, so as to get ${ }^{25}$

$$
\left[\frac{\sum_{i=0}^{h} i^{n} e}{h^{n}(h+1) e}\right]_{h=\infty}=\left[\frac{\sum_{i=0}^{h} i^{n}}{h^{n}(h+1)}\right]_{h=\infty} \frac{1}{n+1} .
$$

Then, he applies this new general result to parabolas of any integer positive order, by concluding that if the curve AO is a parabola of order $n^{26}$, then each surface AOT is to the corresponding parallelogram ADOT as 1 to $n+1$, and consequently each surface ADO is to the same parallelogram as $n$ to $n+1^{27}$.

then all equal to $\frac{k}{\infty}$, and the elements composing this surface (or the sides of these elements parallel to TO) are as $\left(\frac{0}{\infty} k\right)^{n},\left(\frac{1}{\infty} k\right)^{n},\left(\frac{2}{\infty} k\right)^{n}, \ldots,\left(\frac{\infty}{\infty} k\right)^{n}$, with $n=2$ or $n=3$. Hence, for any integer non-negative number $i$, the $i$-th element (or its side parallel to TO) is to $\left(\frac{i}{\infty} k\right)^{n}$ as $l$ is to $k^{n}$, and is thus equal to $\left(\frac{i}{\infty}\right)^{n} l$. It follows that the ratio of AOT to ADOT is equal to $\left[\frac{\sum_{i=0}^{h}\left(\frac{i}{h}\right)^{n} l}{(h+1) l}\right]_{h=\infty}$ or $\left[\frac{\sum_{i=0}^{h} i^{n} e}{(h+1) h^{n} e}\right]_{h=\infty}$, for $e=\frac{1}{h^{n}} l$. Taken as such, this argument involves the multiplication on segments. But it is clear how it could be rephrased to avoid any appeal to such an operation. Indeed, to reach the conclusion, it is enough to take the $i$-th element composing AOT (or its side parallel to TO) to be equal to $\left(\frac{i}{\infty}\right)^{n} l$, which is easy to argue for with the only resources of the theory of proportions.

${ }^{23}$ The basic idea here is to consider a cone or a pyramid as being generated by a triangle and formed by an infinite number of circles or polygons which are respectively to each other in duplicate ratio of the elements composing this triangle. As the latter are arithmetic proportional, the former are to each other in duplicate ratio of arithmetic proportionals and proposition XXI applies.

${ }^{24}$ The crucial remark seems here to be that the sectors TMT of an Archimedean spiral (fig. 3) are to each other as the corresponding right-angled similar triangles, that is, in duplicate ratio of the arithmetic proportionals MT. Differently than in the case of rectification, this entails no error in the case of quadrature, since the replacement of sectors TMT with the corresponding triangles is area-preserving though not being length-preserving ([20], 123).

${ }^{25}$ Though Wallis explicitly justifies this generalisation inductively, the way as he writes the sums of the series $\sum_{i=0}^{h}$ for calculating these sums for any value of $n$ like that exposed by Pascal in the Potestatum numericarum summa: cf. [36], and, for a modern reconstruction [14], 82-84 and [34], 197-200.

${ }^{26}$ According to the condition stated in footnote (19), this means that if the differences TT are all equal to each other and to the first of segments AT, then the segments TO are to each others in $n$-plicate ratio of arithmetic proportionals. Hence, if $\mathrm{AT}_{i}$ and $\mathrm{AT}_{j}$ (where $i$ and $j$ are integer positive indexes) are so taken that $\mathrm{AT}_{i}: \mathrm{AT}_{j}=i: j$ - to the effect that $\beta=\frac{j^{n}}{i^{n-1}}$ - the corresponding segments $\mathrm{T}_{i} \mathrm{O}_{i}=\mathrm{AD}_{i}$ and $\mathrm{T}_{j} \mathrm{O}_{j}=\mathrm{AD}_{j}$ are such that $\mathrm{T}_{i} \mathrm{O}_{i}: \mathrm{T}_{j} \mathrm{O}_{j}=i^{n}: j^{n}$.

${ }^{27}$ Of course, this could be justified through the same argument advanced in footnote (22), by taking $n$ to be any positive integer. In the scholium to proposition XLV ([51], 36-37, and [58], 43-44), Wallis also argues that an analogous generalisation is possible with respect to spirals, so as to get the rectification and quadrature of non-Archimedean spirals where the segments MT (fig. 3) are to each other in $n$-plicate $(n=2,3, \ldots)$ ratio of the corresponding angles TMT. 
These are quite general results. In modern terms, the first of them provides the quadrature of any curve expressed in a Cartesian system of coordinates by an equation of the form $y=\alpha x^{n}$ (where $\alpha$ is any constant coefficient and $n$ any integer positive exponent). One should not believe, however, that this result is somehow analogous to the following:

$$
\int_{0}^{x_{0}} \alpha x^{n} d x=\frac{1}{n+1} \alpha x_{0}^{n+1} .
$$

This is not only because Wallis is quite far from any notion close to that of integral, but also and overall since his result provides no measure for the surfaces AOT or for their area. Despite his considering areas of triangles, in proposition III of the De sectionibus conicis, here he limits himself to calculate the ratio of these surfaces and the corresponding parallelograms ADOT, or better to show that

$$
\text { AOT : } \mathrm{ADOT}=1: n+1,
$$

provided that AO be a parabola of order $n$.

One could think that the passage from this proportion to the equality

$$
\mathrm{AOT}=\frac{(\mathrm{AT})(\mathrm{TO})}{n+1} \sin (\widehat{\mathrm{ADO}})
$$

is trivial. But this is not so. What is trivial is the passage from this proportion to the equality

$$
\mathrm{AOT}=\frac{1}{n+1} \mathrm{ADOT}
$$

not that from this last equality to the equality (12), since this last passage requires not only the admission of multiplication on segments, but also the supposition that the result of such a multiplication can be equated to a plane figure, or provide a measure of it. In Descartes' geometric algebra, this last admission is correct only if such a measure is identified with a segment (since in this algebra the product of two segments is a segment, in turn). In Viète's formalism this admission is natural, instead, but Wallis makes no effort to explain how to use this formalism to express areas.

The consequence of Wallis's limiting himself to state proportion (11) is mathematically relevant. For this proportion provides no base for a linear algorithm of areas. It merely provides an instruction for constructing (by rule and compass) a polygon equal to AOT. This is what Wallis himself says in the scholium to proposition XLV ([51], 36, and [58], 43):

And indeed, by this means, for innumerable curvilinear figures, it will be possible to construct equal rectilinear ones. That which Archimedes (and other after him) showed (with greatest admiration) in the parabola alone, we have now shown for parabolas of any power whatever.

Probably Wallis would have restrained his pride if he had been aware that a few years before Cavalieri had offered an analogous result in the fourth of his Exercitationes geometric ${ }^{28}$. But,

\footnotetext{
${ }^{28}$ Cf. [7], 243-319, especially prop. XXIII, 279-280. On this matter, cf. chapter ???, pp. ??? of the present book and [16], ??Dept. Fil. BO: 13 A 001025??, and [1], 345-347.
} 
also in this case, what is more relevant is not the result as such, but the way it is reached. Since, once more, this immediately suggests a possible generalisation. This goes as follows.

In proposition XLIV, the results expressed by equality (9) for $n=0,1, \ldots, 10$ are embraced in a single table that is taken to be indefinitely continued. In this table, the series $\sum_{i=0}^{h} i^{n} e$ for $n=0,1,2, \ldots$-which Wallis calls respectively 'series of equals', 'series of firsts', 'series of seconds', ...-are respectively related with the denominator of the ratio they have with the corresponding series of equals (namely $\sum_{i=0}^{h} h^{n} e=h^{n}(h+1) e$ ), if $h=\infty$ : each row of this table associates an integer number $n$ understood as the value of the exponent characterising a series like $\sum_{i=0}^{h} i^{n} e$, with its successor $n+1$, understood as the value of the denominator of the ratio $\frac{1}{n+1}$ which is said to be equal to $\left[\frac{\sum_{i=0}^{h} i^{n} e}{h^{n}(h+1) e}\right]_{h=\infty}$. Propositions XLVI-XLVII ([51], 37-39, and [58], 45) show that what Wallis takes to be relevant in this table is that the two sequences it involves are both arithmetic progressions. He argues, indeed, that it is enough to know a couple of items that this table associates to each other, in order to get the item associated to any other item: if the number $\iota$ is associated to the number $\kappa$, then the number $\lambda$ has to be associated to the number $\kappa-\iota+\lambda$.

This is doubly relevant. Geometrically, this allows to conclude - as Wallis does in propositions XLVIII-L ([51], 39-41, and [58], 46-47) - that the conoids or pyramids of axis AT generated by the the surfaces AOT delimited by any parabola of order $n$ are to a cylinder or prism with the same base and the same altitude as 1 to $2 n+1$, since, whatever integer non-negative number $n$ might be, the table associates $n+1$ to it, and then $2 n+1$ to $2 n$. Arithmetically (and, by far more importantly), this suggests an obvious way for interpolating the table: it is enough to require that the interpolation be such to conserve the property of associating to each other two arithmetic progressions. From this condition follows that the interpolated table has to associate to any non-negative rational number $\frac{p}{q}$ the other non-negative rational number $\frac{p+q}{q}$. This is what Wallis states (without any other justification) ${ }^{29}$ in propositions LI, LIII-LIV, and LVIII-LIX ([51], 41-44, 46-48 and [58], 47-49, 51-53), the last of which exhibits the following new table:

\footnotetext{
${ }^{29} \mathrm{~A}$ possible further justification that remains close to Wallis's conception is suggested in [30], 351-353.
} 


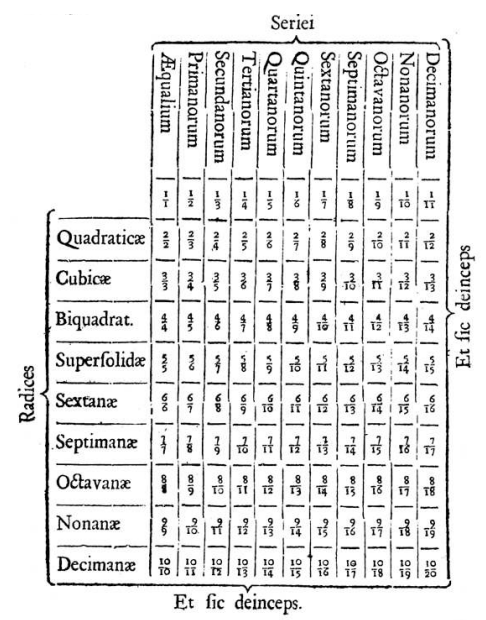

or

\begin{tabular}{l|cccccc}
$q \backslash p$ & 0 & 1 & 2 & 3 & 4 & $\ldots$ \\
\hline 1 & $\frac{1}{1}$ & $\frac{1}{2}$ & $\frac{1}{3}$ & $\frac{1}{4}$ & $\frac{1}{5}$ & $\ldots$ \\
2 & $\frac{2}{2}$ & $\frac{2}{3}$ & $\frac{2}{4}$ & $\frac{2}{5}$ & $\frac{2}{6}$ & $\ldots$ \\
3 & $\frac{3}{3}$ & $\frac{3}{4}$ & $\frac{3}{5}$ & $\frac{3}{6}$ & $\frac{3}{7}$ & $\ldots$ \\
4 & $\frac{4}{4}$ & $\frac{4}{5}$ & $\frac{4}{6}$ & $\frac{4}{7}$ & $\frac{4}{8}$ & $\ldots$ \\
$\vdots$ & $\vdots$ & $\vdots$ & $\vdots$ & $\vdots$ & $\vdots$ & $\ddots$
\end{tabular}

It is easy to see that what this table states is that

$$
\left[\frac{\sum_{i=0}^{h} \sqrt[q]{i^{p}} e}{\sqrt[q]{h^{p}}(h+1) e}\right]_{h=\infty}=\left[\frac{\sum_{i=0}^{h} \sqrt[q]{i^{p}}}{\sqrt[q]{h^{p}}(h+1)}\right]_{h=\infty}=\frac{q}{p+q}
$$

for any integer non-negative number $p$ and any integer positive number $q$.

Wallis's argument to get this last result is certainly not a proof. Still, once this result is applied to geometry, so as to get the quadrature of appropriate curves, it becomes confirmed by its agreement with the quadratures derived from equality (9). It is so because, by reasoning as before, from equality (16), it follows that, if curve $\mathrm{AO}$ is a parabola of order $n(n=1,2, \ldots)$, then each surface ADO is to the corresponding parallelogram ADOT as $n$ to $n+1$, and then each surface AOT is to the same parallelogram as 1 to $n+1^{30}$, as stated above. This is what Wallis remarks in propositions LV-LVII and the corresponding scholium ([51], 44-46 and [58], 50-51).

\footnotetext{
${ }^{30}$ The implicit argument is obvious. Instead of supposing that de differences TT are all equal to each other and to the first of segments AT, one can suppose that the differences DD are all equal to each other and to the first of $A D$, and remark that the segments $\mathrm{DO}$ are then to each other in $\frac{1}{n}$-plicate ratio of arithmetic proportionals. Hence, for any pair of segments $\mathrm{AD}_{i}$ and $\mathrm{AD}_{j}$ (with $i$ and $j$ integer positive indexes) the corresponding pair of segments $\mathrm{D}_{i} \mathrm{O}_{i}=\mathrm{AT}_{i}$ and $\mathrm{D}_{j} \mathrm{O}_{j}=\mathrm{AT}_{j}$ are such that
}

$$
\text { if } \quad \mathrm{AD}_{i}: \mathrm{AD}_{j}=i: j \text { then } \mathrm{D}_{i} \mathrm{O}_{i}: \mathrm{D}_{j} \mathrm{O}_{j}=i: \alpha_{1}=\alpha_{1}: \alpha_{2}=\ldots=\alpha_{n-1}: j
$$


But, of course, equality (16) has also new geometrical consequences. These are stated in propositions LXI-LXIII ([51], 49-51 and [58], 54-55): from this equality, the quadrature of any parabola of any composed order $\frac{p}{q}$ (for whatever positive integer $p$ and $q$ ), and the cubature of the corresponding conoids and pyramids can be obtained. Paraboloids of order $\frac{p}{q}$ are curves like $\mathrm{AO}$ endowed with an axe AD and such that, if $\mathrm{AT}$ is the tangent at the vertex $\mathrm{A}^{q}$, for any pair of points $T$ and $T$ taken on this tangent, the corresponding pair of segments $T O$ and TO parallel to $\mathrm{AD}$ are in $\frac{p}{q}$-plicate (or $p$-plicate sub- $q$-plicate) ratio of the corresponding pair of segments DO and DO parallel to $\mathrm{AT}^{31}$. By applying to these curves the same argument as before, one gets that each surface AOT is to the corresponding parallelogram ADOT as $q$ to $p+q$, and each surface ADO is to this same parallelogram as $p$ to $p+q^{32}$. In the same way, the conoids or pyramids of axis AT generated by the the surfaces AOT, and the conoids or pyramids of axis AD generated by the the surfaces ADO are to a cylinder or prism with the same base and the same altitude as 1 to $\frac{2 p+q}{q}$, and as 1 to $\frac{2 q+p}{p}$, respectively.

These last geometric results are much more general than those deriving from equality (9), since they respectively provide the quadrature of any curve expressed, relatively to a Cartesian system of coordinates, by an equation of the form $y=\alpha x^{\frac{p}{q}}$ (where $\alpha$ is any constant coefficient and $\frac{p}{q}$ any rational positive exponent), and the cubature of the corresponding conoids and pyramids. Also in this case, Wallis makes perfectly explicit the geometric content of his result, by observing, in the scholium to proposition LXI ([51], 50 and [58], 54), that, thanks to it "other curved figures [...] are reduced to equal rectilinear figures" ${ }^{33}$. On the other hand, insofar as he has no difficulty in expressing this same result trough an equality like AOT $=\frac{q}{p+q}$ ADOT, he may easily ground on it the squaring of any portion TOOT of the surfaces AOT and the determination of the ratio between any two such surfaces. This he does in propositions LXVI and LXV, respectively ([51], 53-54 and [58], 56-57) $)^{34}$.

from which it follows that

$$
\text { if } \quad \mathrm{AD}_{i}: \mathrm{AD}_{j}=i: j \text { then } \quad \mathrm{D}_{i} \mathrm{O}_{i}: \mathrm{D}_{j} \mathrm{O}_{j}=i: \sqrt[n]{i^{n-1} j}=\sqrt[n]{i}: \sqrt[n]{j} \text {, }
$$

so that the ratio of ADO and ADOT is equal to $\left[\frac{\sum_{i=0}^{h} \sqrt[n]{i}}{\sqrt[n]{h}(h+1)}\right]_{h=\infty}=\frac{n}{n+1}$. An analogous argument also allows to conclude that the conoids or pyramids of axis AD generated by the surface ADO are to a cylinder or prism with the same base and the same height as $n$ to $n+2$, as Wallis remarks in proposition LX ([51], 48-49 and [58], 53).

${ }^{31}$ This means that for any pair of segments $\mathrm{AT}_{i}$ and $\mathrm{AT}_{j}$ (with $i$ and $j$ integer positive indexes) the corresponding pair of segments $\mathrm{T}_{i} \mathrm{O}_{i}=\mathrm{AD}_{i}$ and $\mathrm{T}_{j} \mathrm{O}_{j}=\mathrm{AD}_{j}$ are such that

$\mathrm{T}_{i} \mathrm{O}_{i}=\mathrm{T}_{j} \mathrm{O}_{j}=\alpha_{0}: \beta_{1}=\beta_{1}: \beta_{2}=\beta_{2}=\ldots=\beta_{q-1}: \gamma \quad$ if and only if $\quad \mathrm{AT}_{i}: \mathrm{AT}_{j}=\alpha_{0}: \alpha_{1}=\alpha_{1}: \alpha_{2}=\ldots=\alpha_{p-1}: \gamma$ where $\alpha_{k},(k=0, \ldots, p-1), \beta_{k},(k=0, \ldots, q-1)$ and $\gamma$ are appropriate quantities.

${ }^{32}$ Since, if the differences TT are all equal to each other and to the first of segments AT, then the segments TO are to each other in $\frac{p}{q}$-plicate ratio of arithmetic proportionals. Hence, according to the condition stated in footnote (31), if $\mathrm{AT}_{i}$ and $\mathrm{AT}_{j}$ (with $i$ and $j$ integer positive indexes) are so taken that $\mathrm{AT}_{i}: \mathrm{AT}_{j}=i: j$ - to the effect that $\gamma=\frac{j^{p}}{i^{p-1}}$, then the corresponding segments $\mathrm{T}_{i} \mathrm{O}_{i}=\mathrm{AD}_{i}$ and $\mathrm{T}_{j} \mathrm{O}_{j}=\mathrm{AD}_{j}$ are such thatAD $\mathrm{AD}_{j}=i^{\frac{p}{q}}: j^{\frac{p}{q}}$

${ }^{33}$ In propositions LXII-LXIII ([51], 50-51 and [58], 54-55), Wallis makes the same point for the conoids ad pyramids generated by the surfaces AOT and ADO.

${ }^{34}$ Let $\mathcal{T}_{1}$ and $\mathcal{T}_{2}$ be two surfaces AOT $\left(\mathcal{T}_{1}<\mathcal{T}_{2}\right)$ delimited by the same curve, and $\mathcal{P}_{1}$ and $\mathcal{P}_{2}$ the corresponding parallelograms ADOT. It follows that $\frac{\mathcal{T}_{1}}{\mathcal{P} 1}=\frac{\mathcal{T}_{2}}{\mathcal{P}_{2}}=\frac{q}{p+q}$, and thus: $\mathcal{T}_{2}-\mathcal{T}_{1}=\frac{q}{p+q}\left(\mathcal{P}_{2}-\mathcal{P}_{1}\right)$. Hence, if one take $\mathcal{T}_{2}-\mathcal{T}_{1}$, and $\mathcal{P}_{1}-\mathcal{P}_{2}$ to be respectively the the surface TOOT included between $\mathcal{T}_{1}$ and $\mathcal{T}_{2}$ and the corresponding gnomon DDOTTO, one gets TOOT $=\frac{q}{p+q}$ DDOTTO. In the same way, if $\mathcal{T}_{1}$ and $\mathcal{T}_{2}$ are two surfaces AOT 
Notice that table (14) or (15) displays an arithmetic relation connecting any value of the ratio $\frac{p}{q}$ to the value provided by the rule $\frac{p}{q} \rightarrow \frac{1}{\frac{p}{q}+1}=\frac{q}{p+q}$. This remark-which Wallis makes in proposition LXIV ([51], 52-53 and [58], 56) - is much less obvious that it can appear at first glance. It makes manifest, indeed, that the only information relative to the series $\sum_{i=0}^{h} \sqrt[q]{i^{p}} e$ that is relevant for calculating the ratio it has, if continued to the infinite, with the corresponding series of equals $\sum_{i=0}^{h} \sqrt[q]{h^{p}} e=(h+1) \sqrt[q]{h^{p}} e$ is the value of the ratio $\frac{p}{q}$. As Wallis explicitly says, this latter ratio can thus be understood as an "index" and his table as the expression of a relation between this index and the value of the ratio $\left[\frac{\sum_{i=0}^{h} \sqrt[q]{i^{p}} e}{\sqrt[q]{h^{p}}(h+1) e}\right]_{h=\infty}$. Using a convenient notation (which is not Wallis's, of course), this understanding can be rendered by rewriting equality (16) as follows:

$$
\frac{\sum_{r}}{\bigcup_{r}}=\frac{1}{r+1},
$$

where $r$ is a non-negative rational index and ' $\sum_{r}$ ' and ' $\bigcup_{r}$ ' denote, respectively, the series $\sum_{i=0}^{h} i^{r} e$ and $\sum_{i=0}^{h} h^{r} e=h^{r}(h+1) e$, continued to the infinite. Now, once these series and the ratios $\frac{\sum_{r}}{\mathrm{U}_{r}}$ and $\frac{1}{r+1}$ are conceived as arithmetic objects associated to the index $r$, this index may be assumed to be any non-negative rational number. This is what Wallis remarks at the and of his proof of proposition LXIV ${ }^{35}$ :

If the index is supposed irrational, for example $\sqrt{3}$, the ratio will be as 1 to $1+\sqrt{3}$.

Geometrically speaking, the consideration of irrational values of $r$ is useless, however. What is relevant is rather the possibility of composing different series $\sum_{r}$ and $\bigcup_{r}$ through multiplication and division, as Wallis explicitly says in propositions LXXIII and LXXXI ([51], 58-59, 63-64, and [58], 61, 65-66, respectively):

If any two series (or also more) were respectively multiplied to each other (that is, the first term of one of them by the first of the other, the second by the second, etc.), it would result another series of the same kind, whose index will result from the addition of the indices of the multiplied [series].

If all the terms of a series are respectively divided by the terms of another series, the quotients will form another series, whose index is found by subtracting the indexes of the dividing series from the index of the divided series $[\ldots]$.

delimited by the different curves, their ratio is equal to $\frac{\chi_{2}}{\chi_{1}} \frac{\mathcal{P}_{1}}{\mathcal{P}_{2}}$, where $\frac{1}{\chi_{1}}$ and $\frac{1}{\chi_{2}}$ are respectively the values of $\frac{q}{p+q}$ relative to the two curve delimiting $\mathcal{T}_{1}$ and $\mathcal{T}_{2}$. Propositions LXVII-LXXII ([51], 54-58 and [58], 58-61) present then some obvious consequences of these results.

${ }^{35}$ Wallis's point here is clearly that $r$ can be viewed as a mere index, independently of any arithmetic or geometric meaning of the corresponding series and ratios. But his reasoning also suggests the possibility of defining irrational powers by interpolation on rational ones, as it will rapidly become usual in the second half of 17 th century. 
Using the previous notation, these definitions can be rendered as follows:

$$
\begin{aligned}
& \sum_{r} \cdot \sum_{s}=\sum_{r+s} \quad \bigcup_{r} \cdot \bigcup_{s}=\bigcup_{r+s} \\
& \frac{\sum_{r}}{\sum_{s}}=\sum_{r-s} \quad \frac{\bigcup_{r}}{\bigcup_{s}}=\bigcup_{r-s}
\end{aligned}
$$

Moreover, as $\sum_{0}=\sum_{i=0}^{h} i^{0} e=(h+1) e$ and $\bigcup_{r}=h^{r}(h+1) e$, one gets $\bigcup_{r}=h^{r} \sum_{0}$, and, because of this equality and the equality (17):

$$
\begin{gathered}
\bigcup_{r} \cdot \sum_{s}=\frac{1}{s+1} \bigcup_{r} \cdot \bigcup_{s}=\frac{1}{s+1} \bigcup_{r+s} \\
\frac{\sum_{r}}{\bigcup_{s}}=\frac{1}{h^{s}} \sum_{r}=\frac{1}{r+1} \frac{\bigcup_{r}}{h^{s}}=\frac{1}{r+1} \frac{\bigcup_{r} \sum_{0}}{\bigcup_{s}}=\frac{1}{r+1} \bigcup_{r-s} \\
\frac{\bigcup_{r}}{\sum_{s}}=h^{r} \sum_{-s}=\bigcup_{r} \frac{\sum_{-s}}{\sum_{0}}=\bigcup_{r} \sum_{-s}=\frac{1}{1-s} \bigcup_{r-s}
\end{gathered}
$$

The geometric interpretation of multiplication is quite natural. By multiplying a series $\sum_{r}$ by another series $\sum_{s}$ or $\bigcup_{s}$, one gets a series whose terms are to each other as rectangles whose sides DO and DM, or DO and DF (fig. 5) are to each other as the terms of these series and compose then a solid AMNOD or AEFGOD. In the same way, by multiplying a series $\bigcup_{r}$ by another series $\bigcup_{s}$, one gets a series of equals whose terms are proportional to the equal rectangles that compose a prism (whose sides are proportional to the terms of these series). Wallis clarifies this matter through examples in propositions LXV-LXXX ([51], 53-63, and [58], 56-65).
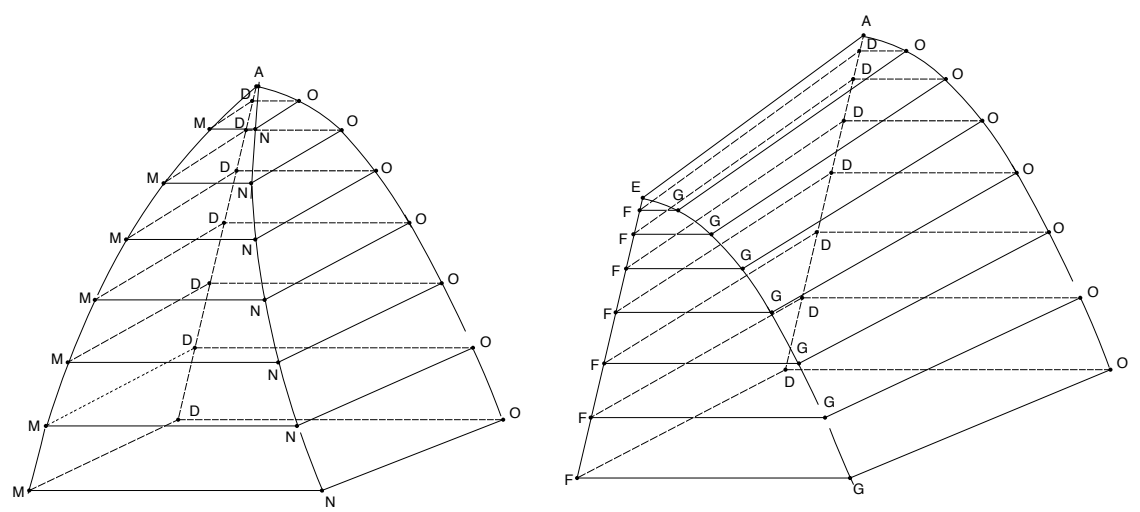

Fig. 5

The geometric interpretation of division is much more problematic, overall if, following Wallis, no restriction is made on the values of the indexes of the relevant series, and negative indexes are thus allowed. In the Euclidean parlance, one says that an area is applied to a given segment $\alpha$ when a plane figure $\mathcal{G}$ of a specified kind which is equal to another given plane figure $\mathcal{F}$ is constructed on this segment. The area of $\mathcal{F}$-or better $\mathcal{F}$ itself taken only for its quantitative properties - is then applied to $\alpha$ by constructing $\mathcal{G}^{36}$. In the simplest case, $\mathcal{G}$ is required to be a

\footnotetext{
${ }^{36}$ The locus classicus for this terminology is proposition I.44 of the Elements. On this matter, cf., for example, Heath's comment to this proposition: [15], I, 343-345.
} 
rectangle. In this case, to say that (the area of) $\mathcal{F}$ is applied to $\alpha$ means that another segment $\beta$ is constructed in a such a way that the rectangle $\mathcal{R}(\alpha, \beta)$ of sides $\alpha$ and $\beta$ is equal to $\mathcal{F}$. By using this parlance, Wallis says that a solid figure, whose elements are some plane figures, "is respectively applied" to a plane figure, whose elements are some segments (propositions LXXXIII and LXXXV: [51], 65-66 and [58], 66-67) to mean that another plane figure have been constructed (or more generally determined) in such a way that its elements are other segments such that the rectangles whose sides are respectively these latter segments and the former ones are respectively equal to the elements of the solid figure. Wallis also expresses the same idea (for example in propositions LXXXVIII-XC: [51], 68-70 and [58], 70-71), by saying that "the planes" of the solid figure are applied to "the straight lines" of the plane one. Using a convenient notation (which is not Wallis's), one could say that a solid figure $\mathfrak{X}$-whose elements are some plane figures $\mathcal{X}_{i}$-is respectively applied to a plane figure $\mathcal{Y}$ - whose elements are some segments $y_{i}$ - (or the planes $\mathcal{X}_{i}$ of $\mathfrak{X}$ are applied to the segments $y_{i}$ of $\mathcal{Y}$ ), to mean that another plane figure $\mathcal{Z}$ has been constructed (or more generally determined) whose elements are other segments $z_{i}$ such that the rectangles $\mathcal{R}\left(y_{i}, z_{i}\right)$ of sides $y_{i}$ and $z_{i}$ are respectively equal to the plane figures $\mathcal{X}_{i}$. Wallis also says (in propositions LXXXIV, LXXXVI, and LXXXVIII: [51], 64-69 and [58], 67-70) that the "third proportionals" to the respective straight lines of two planes figures are taken, to means that a third plane figure has been constructed (or more generally determined) in such a way that the rectangles whose sides are respectively the elements of this third figure and those of the first one are equal to the squares whose sides are the elements of the second future. In other terms, taking the third proportionals to the respective straight lines of two planes figures $\mathcal{Y}$ and $\mathcal{X}$, whose elements are respectively some segments $y_{i}$ and $x_{i}$, is the same as constructing (or more generally determining) a third plane figure $\mathcal{Z}$ whose elements $z_{i}$ are such that the rectangles $\mathcal{R}\left(y_{i}, z_{i}\right)$ with sides $y_{i}$ and $z_{i}$ are respectively equal to the squares $\mathcal{S}\left(x_{i}\right)$ whose sides are the elements $x_{i}$ of $\mathcal{X}$.

By using this terminology, Wallis provides the geometric interpretation of division on his series as follows.

In propositions LXXXIII-LXXXVI ([51], 65-67 and [58], 66-68), he offers some examples illustrating the case in which the index $r-s$ of the quotient of two series is non-negative. Some of them (advanced in proposition LXXXV) concern a parabolic pyramid (whose elements are to each other as the terms the series $\sum_{1}$ ) respectively applied to a triangle (whose elements are also to each other as the terms the series $\sum_{1}$ ), to a parallelogram (whose elements are to each other as the terms the series $\sum_{0}$ ), to a parabolic surface (whose elements are to each other as the terms the series $\sum_{\frac{1}{2}}$ ), and to a cubic surface (whose elements are to each other as the terms the series $\sum_{\frac{1}{3}}$ ), so as to get, respectively, a parallelogram (since $\frac{\sum_{1}}{\sum_{1}}=\sum_{0}$ ), a triangle (since $\frac{\sum_{1}}{\sum_{0}}=\sum_{1}$ ), a parabolic surface (since $\frac{\sum_{1}}{\sum_{\frac{1}{2}}}=\sum_{\frac{1}{2}}$ ), and a surface delimited by a parabola of order $\frac{2}{3}$ (since $\frac{\sum_{1}}{\sum_{\frac{1}{3}}}=\sum_{\frac{2}{2}}$ ). Other examples (advanced in proposition LXXXVI) concern the third proportionals to the respective straight lines of a triangle and a parabolic surface, or to the respective straight lines of a parallelogram and a parabolic surface, or to the respective straight lines of a parabolic surface and a cubic surface, which produce respectively $(i$. $e$., are respectively the elements of) a parallelogram (since $\sum_{1}: \sum_{\frac{1}{2}}=\sum_{\frac{1}{2}}: \sum_{0}$ ), a triangle (since $\sum_{0}: \sum_{\frac{1}{2}}=\sum_{\frac{1}{2}}: \sum_{1}$ ), or a quadric surface (since $\sum_{2}: \sum_{3}=\sum_{3}: \sum_{4}$ ). 
In propositions LXXXVII-CI ([51], 67-74 and [58], 68-75), he considers then the case in which the index $r-s$ of the quotient of two series is negative. His different examples can be accounted for as particular cases of the following general argument.

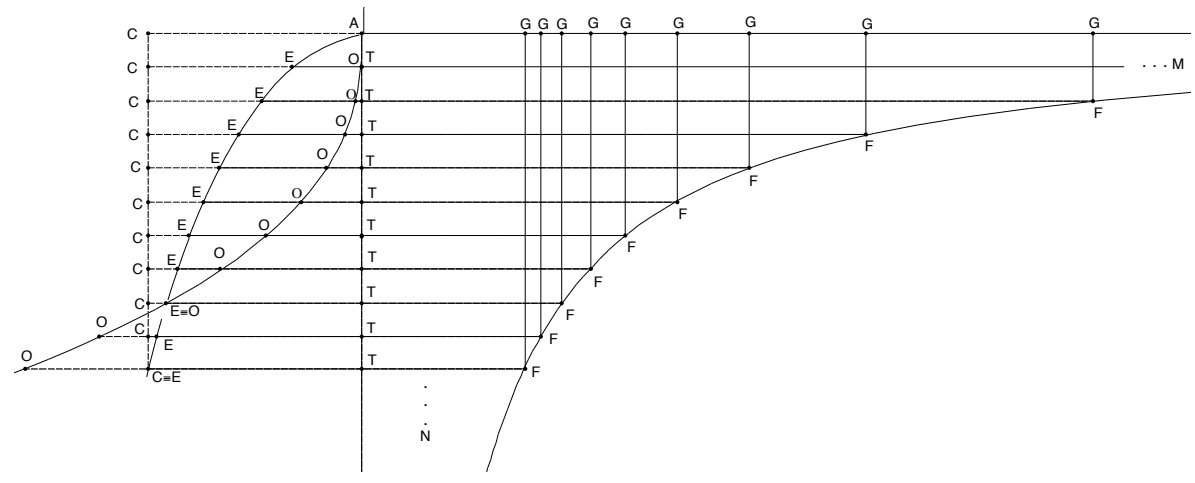

Fig. 6

Let AOT (fig. 6) ${ }^{37}$ any surface delimited by a parabola $\mathrm{AO}$ of any positive rational order $s$. Consider two cases. In the first, let $\mathfrak{X}$ be a solid figure whose elements are to each other as the rectangles $\mathcal{R}\left(\mathrm{ET}, z_{i}\right)$ or $\mathcal{R}\left(\mathrm{CT}, z_{i}\right)$ whose sides are respectively the elements $\mathrm{ET}$ or $\mathrm{CT}$ of a surface AET or a rectangle ACCT and other segments $z_{i}$ so varying that the elements of $\mathfrak{X}$ are to each other as the terms of a series with a rational non-negative index $r$ smaller than $s$. For example, let (like in proposition LXXXVIII) AOT be a triangle and $\mathfrak{X}$ a parallelepiped whose elements are the rectangles $\mathcal{R}(\mathrm{CT}, z)$ where $z$ is a constant segment so that $s=1$ and $r=0$, or let (like in proposition LXXXIX) AOT be a cubic surface, AET a triangle, and $\mathfrak{X}$ a pyramid whose elements are the squares $\mathcal{S}(\mathrm{ET})$ with sides ET, so that $s=3$ and $r=2$. Suppose then that $\mathfrak{X}$ be respectively applied to AOT (or the planes of $\mathfrak{X}$ are applied to the straight lines of AOT). The result is a plane figure whose elements are to each other as the elements of the series $\sum_{r-s}=\frac{\sum_{r}}{\sum_{s}}$, where $r-s$ is a negative index. In the second case, avoid to consider any solid figure, and suppose that the segments ET be to each other as the terms of a series $\sum_{\frac{r}{2}}$ where $r$ is any non-negative rational index smaller than $s$, and that the third proportionals to the respective straight lines of the surfaces AOT and AET, or of the surface AOT and the rectangle ACCT, be taken. For example, let-like in proposition LXXXVIII, again-AOT be a triangle and suppose that the third proportionals to the respective straight lines of AOT and the rectangle ACCT be taken, so that $s=1$ and $\frac{r}{2}=0$. The result is a third plane figure whose elements are to each other are the elements of the series $\sum_{r-s}=\frac{\left(\sum_{\frac{r}{2}}\right)^{2}}{\sum_{s}}$, where $r-s$ is a negative index (of course, in the case where the third proportionals are those to the respective lines of AET and ACCT, $r$ is 0 an $r-s=-s$ ).

Suppose now that $r-s=-\frac{p}{q}$, where $p$ and $q$ are positive integers. The resulting plane figure whose elements are to each other as the elements of the series $\sum_{r-s}$ is then a rectangular hyperboloid of order $\frac{p}{q}$ with asymptotes AT and AG. This is a curve MN such that any pair of

\footnotetext{
${ }^{37}$ For reasons of simplicity, uniformity and convenience, I change Wallis's letters in the figure and use a unique figure to account for different propositions to which different Wallis's figures are appended. I also add the letters ' $N$ ' and ' $M$ ' to designate two points at the infinity which do not occur in Wallis's figures.
} 
points $T$ and $T$ taken on the former of these asymptotes are such that the corresponding pair of segments TF and TF parallel to AG are in the inverse $\frac{p}{q}$-plicate ratio of the corresponding pair of segments GF and GF parallel to $A T^{38}$. Let then $F_{1}$ and $F_{2}$ be any two points $F$ on any curve like that, the latter of which is either identical with the former or closer to $M$. Let also $T_{1}$ and $T_{2}$ be respectively the orthogonal projections of $F_{1}$ and $F_{2}$ on AT, and $G_{1}$ and $G_{2}$ the orthogonal projections of $F_{1}$ and $F_{2}$ on $A G$. One can then associate to $F_{1}$ the rectangle $A T_{1} F_{1} G_{1}$ whose elements are all equal and parallel to $T_{1} F_{1}$, and to $F_{2}$ both an element of this rectangle, namely, the initial portion of $T_{2} F_{2}$ equal to $T_{1} F_{1}$, and an element of the infinite surface $A T_{1} F_{1} M$, namely the whole segment $T_{2} F_{2}$. Hence, if equality (17) is extended to the case of negative rational indexes and an argument analogous to that leading to the quadrature of higher order parabolas is applied, one gets ${ }^{39}$

$$
\frac{\text { ATFM }}{\text { ATFG }}=\frac{\sum_{-\frac{p}{q}}}{U_{-\frac{p}{q}}}=\frac{q}{q-p},
$$

which holds, of course, for any point $F$ taking the role of the point $F_{1}$ in the previous argument.

This result Wallis states in propositions CII-CV ([51], 76-79 and [58], 76-79). In these same propositions, he also argues that the ratio $\frac{\text { ATFM }}{\text { ATFG }}$ is then positive if $\frac{p}{q}<1$, infinite if $\frac{p}{q}=$ 1 (so that curve NM is an ordinary hyperbole), and "more than an infinite one, that is, as that which a positive number may be supposed to have to a negative number" 40 , if $\frac{p}{q}>1$. None of these conclusions seems to astonish him. Whereas he justifies the first one (in the scholium to proposition CVII: [51], 83-84 and [58], 81-82) by referring to Torricelli's proof of the equality between the infinite solid generated by the revolution of an hyperbole around one of its asymptotes and a corresponding finite cylinder ${ }^{41}$, he offers no explicit justification for the

\footnotetext{
${ }^{38}$ This means that for any pair of segments $\mathrm{AT}_{i}$ and $\mathrm{AT}_{j}$ (with $i$ and $j$ integer positive indexes) the corresponding pair of segments $\mathrm{T}_{i} \mathrm{~F}_{i}=\mathrm{AG}_{i}$ and $\mathrm{T}_{j} \mathrm{~F}_{j}=\mathrm{AG}_{j}$ are such that

$\mathrm{T}_{j} \mathrm{~F}_{j}: \mathrm{T}_{i} \mathrm{~F}_{i}=\alpha_{0}: \beta_{1}=\beta_{1}: \beta_{2}=\beta_{2}=\ldots=\beta_{q-1}: \gamma \quad$ if and only if $\quad \mathrm{AT}_{i}: \mathrm{AT}_{j}=\alpha_{0}: \alpha_{1}=\alpha_{1}: \alpha_{2}=\ldots=\alpha_{p-1}: \gamma$ where $\alpha_{k},(k=0, \ldots, p-1), \beta_{k},(k=0, \ldots, q-1)$ and $\gamma$ are appropriate quantities. If $p=q=1$, the curve is of course an ordinary hyperbola since, in this case,

$$
\mathrm{T}_{j} \mathrm{~F}_{j}: \mathrm{T}_{i} \mathrm{~F}_{i}=\mathrm{AT}_{i}: \mathrm{AT}_{j} .
$$

It is then obvious that, whatever $i$ and $j$ might be, the rectangle with sides $\mathrm{AT}_{i}$ and $\mathrm{T}_{i} \mathrm{~F}_{i}$ is equal to that with sides $\mathrm{AT}_{j}$ and $\mathrm{T}_{j} \mathrm{~F}_{j}$, as remarked by Wallis in proposition XCIV.

${ }^{39}$ If the differences TT are all equal to each other and to the first of segments AT, then the segments TF are to each others in the inverse $\frac{p}{q}$-plicate ratio of arithmetic proportionals. Hence, according to the condition stated in footnote (38), if $\mathrm{AT}_{i}$ and $\mathrm{AT}_{j}$ (with $i$ and $j$ integer positive indexes) are so taken that $\mathrm{AT}_{i}: \mathrm{AT}_{j}=i: j$ - to the effect that $\gamma=\frac{j^{p}}{i^{p-1}}$, then the segments $\mathrm{T}_{i} \mathrm{~F}_{i}=\mathrm{AG}_{i}$ and $\mathrm{T}_{j} \mathrm{~F}_{j}=\mathrm{AF}_{j}$ are such that $\mathrm{T}_{j} \mathrm{~F}_{j}=\mathrm{T}_{i} \mathrm{~F}_{i}=i^{\frac{p}{q}}: j^{\frac{p}{q}}$, i. e. $\mathrm{T}_{j} \mathrm{~F}_{j}=\mathrm{T}_{i} \mathrm{~F}_{i}=j^{-\frac{p}{q}}: i^{-\frac{p}{q}}$.

${ }^{40}$ The quote comes from proposition CIV ([51], 78 and [58], 78). In the scholium to proposition CI and in proposition CV ([51], 74-75, 79 and [58], 75-76, 79), Wallis uses the two phrases 'ratio greater than an infinite one [ratio major quam infinita]' and 'ratio more than an infinite one [ratio plusquam infinita]' with the same meaning. Remark that in these phrases, the adjective 'infinite [infinitus]' is not substantivised, to the effect that the translations 'more than infinite' or 'greater than infinite', which are often used to render Wallis's conclusion are not entirely faithful.

${ }^{41}$ Torricelli's result is stated in the treatise De solido hyperbolico acuto, included in his Opera Geometrica appeared in 1644 ([49], part II, 93-143), where it is proved both by indivisibles and by exhaustion. For a discussion of this result and its reception (also considering Wallis' attitude with respect to it), cf., among others, [31] and [25], 129-149.
} 
second conclusion nor for the third one.

Possibly he considered the second conclusion obvious ${ }^{42}$. But it is hard to imagine that this was also the case for the third. This conclusion has been often judged to be quite odd (for example by [6], II, 902, and [44], 43-46). At first glance, proposition CV seems to suggest that Wallis believed possible to justify it by observing that the ratio of two positive numbers is finite - i. e. less than an infinite ratio - and that of a positive number to zero is just infinite - i. e. neither more nor less than an infinite ratio-, and then concluding, by continuity (or analogy), that the ratio of a positive number to a negative one should then be more than an infinite ratio. A better look to what Wallis says in this proposition licenses, however, another interpretation, by far less contentious. Indeed, Wallis's point here is not merely that the ratio of two positive numbers is finite and that of a positive number to zero is infinite, but rather that when the ratio $\frac{\text { ATFM }}{\text { ATFG }}$ is more than an infinite one, the other ratio $\frac{\text { FGAN }}{\text { ATFG }}$ is finite or less than an infinite one, and, more in general, that when one of these ratios is finite, the other is more than an infinite one and vice versa, and when one of them if just infinite, also the other is so. If we denote by ' $\frac{1}{0}$ ' an infinite ratio, Wallis's point is then that:

$$
\begin{gathered}
\frac{\text { ATFM }}{\text { ATFG }}<\frac{1}{0} \quad \text { IFF } \quad \frac{\text { FGAN }}{\text { ATFG }}>\frac{1}{0} \quad \frac{\text { ATFM }}{\text { ATFG }}>\frac{1}{0} \quad \text { IFF } \quad \frac{\text { FGAN }}{\text { ATFG }}<\frac{1}{0} \\
\frac{\text { ATFM }}{\text { ATFG }}=\frac{1}{0} \quad \text { IFF } \quad \frac{\text { FGAN }}{\text { ATFG }}=\frac{1}{0}
\end{gathered}
$$

This recalls the argument Wallis relies on to confirm equality (16) by comparing its geometric consequences to those of equality (9). Any surface FGAN can be taken to be composed by all the segments GF that lies on the left of one of them. Analogously, any rectangle ATFG can be taken to be composed by the portions of these segments equal to the smaller of them. Now, if the differences GG are all equal to each other and to the first of $A G$, then the segments GF are to each other in the inverse $\frac{q}{p}$-plicate ratio of arithmetic proportionals. Hence, from this understanding and equality (20), it follows that the ratio $\frac{\mathrm{FGAN}}{\mathrm{ATFG}}$ is equal to $\frac{p}{p-q}{ }^{43}$. It is thus plausible to think that in Wallis's parlance, saying that the ratio $\frac{\text { ATFM }}{\text { ATFG }}$ is more than an infinite one is a way for stating a rule prescribing that, in squaring the curve $\mathrm{NM}$, one has not to consider this ratio, but the other ratio $\frac{\text { FGAN }}{\text { ATFG }}$ that is, in this case, perfectly finite ${ }^{44}$.

A similar situation also obtains in the case of the application of equality (20) to the cubature of appropriate solids. This is what Wallis shows in propositions CVI and CVII ([51], 79-83

\footnotetext{
${ }^{42}$ Note however that Wallis is unable to draw from this result any conclusion relative to the quadrature of the hyperbole. This is all the more strange that in the dedication of his treatise $([51],[8]$, and [58], 5-6), he mentions Grégoire de Saint-Vincent's Opus Geometricum, where it is proved that if points T are so taken that segments AT forms an arithmetic progression, then the corresponding surfaces TFFT delimited by an hyperbole form a geometrical progression ([10], prop 109). This makes easy, indeed, to conclude that these surfaces are to each other as the natural logarithms of the ratio of these some segments, as stated in 1649 in a pamphlet published under the name of Grégoire's pupil A. A. de Sarasa ([11]; [12]). Wallis's failure to remark this is possibly due to the fact that his method allows no translation apt to bypass the infinity of the surfaces ATFM

${ }^{43}$ Indeed, according to the condition stated in footnote (38), if $\mathrm{AG}_{i}=\mathrm{T}_{i} \mathrm{~F}_{i}$ and $\mathrm{AG}_{j}=\mathrm{T}_{j} \mathrm{~F}_{j}$ (with $i$ and $j$ integer positive indexes) are so taken that $\mathrm{AG}_{j}: \mathrm{AG}_{i}=i: j$ - to the effect that $\gamma=\gamma=\frac{j^{q}}{i^{q-1}}$-, then the corresponding segments $\mathrm{G}_{i} \mathrm{~F}_{i}=\mathrm{AT}_{i}$ and $\mathrm{G}_{j} \mathrm{~F}_{j}=\mathrm{AT}_{j}$ are such that $\mathrm{G}_{j} \mathrm{~F}_{j}: \mathrm{G}_{i} \mathrm{~F}_{i}=i^{\frac{q}{p}}: j^{\frac{q}{p}}, i . e \cdot \mathrm{G}_{j} \mathrm{~F}_{j}: \mathrm{G}_{i} \mathrm{~F}_{i}=j^{-\frac{q}{p}}: i^{-\frac{q}{p}}$.

${ }^{44}$ In modern terms, this is the same as replacing the integral $\int_{0}^{x_{0}} x^{-\frac{p}{q}} d x=\frac{q}{q-p} x_{0}^{\frac{q-p}{q}}$ (where $x_{0}$ is any positive value of $x$ ) with the integral $\int_{x_{0}}^{\infty} x^{-\frac{p}{q}} d x=\frac{q}{p-q} x_{0}^{\frac{q-p}{q}}$, since the ratio $\frac{\frac{q}{p-q} x_{0}^{\frac{q-p}{q}}+x_{0}^{1-\frac{p}{q}}}{x_{0}-\frac{p}{q}}$ is just equal to $\frac{p}{p-q}$.
} 
and [58], 79-81), where he states that the ratio of an "inverse pyramoid or conoid" of axis AT generated by a surface ATFM delimited by any hyperboloid of order $\frac{p}{q}$ to a cylinder or prism with the same base and the same altitude $(i . e$, , the cylinder or prism of axis AT generated by the corresponding rectangle ATFG) is equal to $\frac{q}{q-2 p}$, and is thus finite if $\frac{p}{q}<\frac{1}{2}$, infinite if $\frac{p}{q}=\frac{1}{2}$ and more than an infinite one if $\frac{p}{q}<\frac{1}{2}$.

\section{Squaring the Circle}

The results obtained in the first part of the Arithmetica infinitorum are quite general but have no direct consequence for the squaring-circle problem. In order to tackle this problem with the same method employed for getting them, new arithmetical results concerning new ratios involving the series $\sum_{r}$ and $\bigcup_{r}$ have to be found. This is what Wallis does in the second part of his treatise. The basic idea is as follows.

Let $A B C$ be a quarter of circle of centre $A$ and radius $A B$ (fig. 7). Suppose this radius be divided in an infinity of parts by points $T$. If $B D$ is the diameter of the whole circle, each half-chord TO corresponding to each point $T$ is the mean proportional between $B T$ and TD. As $T D=A B+A T$ and $B T=A B-A T$, the half-chords $T O$ are to each other as the mean proportionals between $A B+A T$ and $A B-A T$. Then, by taking $A B$ to be the greatest value taken by segments AT and supposing that the differences TT be all equal to each other, Wallis concludes that these same half-chords are to each other as the mean proportionals between the terms of two series $\bigcup_{1}+\sum_{1}$ and $\bigcup_{1}-\sum_{1}$, which, according to equalities (18), means that these half-chords are to each other as the terms of a series like $\left(\bigcup_{2}-\sum_{2}\right)^{\frac{1}{2}}$. As the elements of the square $A C E B$ are all equal to $A B$, it follows that (proposition CXXI: [51], 91-92, and [58], 91):

$$
\frac{\mathrm{ACB}}{\mathrm{ACEB}}=\frac{\left(\bigcup_{2}-\sum_{2}\right)^{\frac{1}{2}}}{\bigcup_{1}}
$$

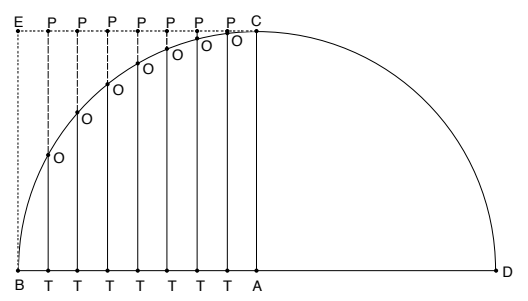

Fig. 7

Through this argument, the circle-squaring problem is reduced to the arithmetic problem of determining the value of the ratio $\frac{\left(\cup_{2}-\sum_{2}\right)^{\frac{1}{2}}}{U_{1}}$. The second part of the Arithmetica infinitorum is mainly devoted to this last problem, which, taken as such, is independent of any geometric consideration and, a fortiori, of any understating and use of indivisibles. There is thus no need here to follow Wallis in the complex argument he offers in order to support his main conclusions, namely that: $i)$ the ratio $\frac{\left(\cup_{2}-\sum_{2}\right)^{\frac{1}{2}}}{U_{1}}$ is equal to the reciprocal of the intermediate term between the first and the second terms of the sequence $\left\{F_{i}\right\}_{i=0}^{\infty}=\{1,2,6,20,70,254, \ldots\}$, where, for any 
$i, F_{i}$ is the $(i+1)$-th figurate number of order $i$ (propositions CLXVIII-CLXIX: [51], 135-137, and [58], 128-130); ii) this intermediate term, and then this ratio, "cannot be expressed in numbers according to any method of notation so far accepted, not even by surds" (scholium to proposition CXC: [51], 174, and [58], 161); iii) such a ratio can however be expressed through the quotient of two infinite products and through a continuos fractions, namely $\prod_{i=1}^{\infty} \frac{(2 i)(2 i+2)}{(2 i+1)^{2}}$ and $\frac{1}{1+\frac{1}{2+\frac{9}{2+\frac{25}{2+\frac{49}{2+\ldots}}}}}$ (proposition CXCI: [51], 178-182, and [58], 164-168).

The negative conclusion (ii) is by far the more relevant outcome of the Arithmetica infinitorum. But Wallis does not stop at it. He does not only add a positive conclusion like (iii), but also goes up to envisage (in the scholium to proposition CXC, too) the possibility of defining new sorts of numbers, together with the usual arithmetic operation on them, by relying on the introduction of new suitable symbols and the interpolation of appropriate series of rationals.

While Wallis's suggestion receives serious treatment neither in the Arithmetica infinitorum, nor in any other of his works, it points to a crucial aspect of his foundational program. The problem it tries to answer-namely, which sort of numbers can express ratios like that of a circle to the square constructed on its diameter - is one that Wallis could not avoid because it is connected to the possibility of expressing geometrical continuity arithmetically. Still, this vague suggestion is the only solution he is able to advance. This failure does not only hinges on his lacking of appropriate mathematical resources. Most importantly, it depends on the fact that the whole framework of his arithmetic treatment of geometric problems fully rests on a primordial intuition of geometric continuity. Wallis's use of indivisibles is nothing, indeed, but an ingenious artifice for making plausible an arithmetic conceptualisation of ratios between continuous magnitudes. Inside this framework, it would have been a petitio principii to define continuity in arithmetic terms. At most, Wallis could have found some clever way for formally expressing ratios between geometric magnitudes that are not amenable to ratios of integers or to rational combinations of surd roots.

The three propositions that close Wallis's treatise (propositions CXCII-CXCIV: [51], 193194 and [58], 178-179) suggest that he was aware of this. They set forth a way of representing geometrically his main results through some "aquabiles" curves. The problematic nature of these curves was already recognised at the time by Wallis as well as by others. In 1655, upon reading the Arithmetica infinitorum, John Pell pointed out that out of Wallis's book "no man will be able to find what he [Wallis] means by 'aequabilis curva"" (cf. British Library: Add Ms 4418, f. 210). There is also evidence that Wallis knew that his use of curves did not really improve his solution of the circle-squaring problem for the very reason that he knew nothing of the nature of his curves. He avowed as much in a letter to Oughtred written just before he sent the Arithmetica infinitorum to the printer. Asking Oughtred's help, he put to him the problems he addressed in Proposiitons 192 to 194 to conclude: "What I aim at is the true nature of the curve line proposed, and how to describe it by points, or how to assign any point thereof required; at least what is the length of the third of those lines parallel to the axis" (Wallis to Oughtred, 28 February 1655: [43], vol. I, 86).

These last three propositions and their accompanying scholium offer a number of deep mathematical problems that we cannot discuss here. We bring them here, if in an incomplete way, only as revealing Wallis's awareness of the problems besetting his arithmetic characterisation of ratios of geometric magnitudes. Wallis assumes the relevant curves to pass through some 
points determined by taking the ordinates of equally distant abscissae in the same ratio to each other as the terms of sequences appearing in his tables, whose interpolation is taken to provide the ratio between a circle and the square on its diameter (i.e. $\frac{\pi}{4}$, in modern notation) or some rational multiples of it. Moreover, he assumes these curves do not move "jerkily from here to there" (which he expresses by terming these curves 'cquabiles'). Then he argues that the relevant ratios are those between appropriate intermediary ordinates of these curves. In other terms, Wallis assumes the existence of some smooth curves introduced ad hoc, and turns them into an instrument for placing the relevant ratios into the geometrical continuum.

Wallis's last argument manifests a peculiar relation between arithmetical and geometrical tools. After having established, in agreement with the method described above (which is already based on a profitable interaction of these tools), that the ratio between a circle and the square on its diameter is provided by interpolating an appropriate sequence of rationals, Wallis appeals to this sequence in order to define a few points of a curve, then supposes that this curve meets appropriate conditions of uniformity and relies on it to hypothetically represent this ratio. This could appear as a devaluation of Wallis's foundational program, which explicitly endorsed the primacy of arithmetic over geometry. Still, even if this were so, the devaluation would not be dramatic, since this program, and, within it, the arithmetic reformulation of the method of indivisibles, did not point to the elimination of geometry from the mathematical arena (with the consequent replacement of it with an appropriate version of arithmetic or with some sort of algebra), but rather to its reformulation on the base of a new and more powerful arithmetic.

\section{Discussing the Notion of Infinite}

As we saw in the foregoing sections, Wallis the mathematician made a (not always successful) effort to avoid committing himself to a specific interpretation of infinitely small elements composing geometrical lines, surfaces and bodies. However, Wallis the polemicist could not avoid the issue with the same tricks he used in his geometrical texts and offers us a fuller view of his metaphysical commitments.

He accurately discussed his own understanding of indivisibles in his response to attacks by Thomas Hobbes ${ }^{45}$. In his Six Lessons to the Professors of Mathematicks (1656: cf. [18], vol. VII, p. 300-301, and also [5] and [42]), Hobbes criticised Wallis's use of infinitesimals with the classical argument that infinitesimals must be either equal to zero or have a finite magnitude. He also criticised Wallis for departing from Cavalierian indivisibles, that is for departing from the view that the indivisibles of lines are points, those of surfaces are lines, and so on, to embrace instead the view that indivisibles are infinitesimally small quantities homogeneous with the whole: "your Indivisibles [...] are supposed to have quantity, that is to say, to be divisibles" ([18], vol. VII, p. 301). Perhaps the most formal and civilised expression of the differences between Hobbes and Wallis is to be found in 1671, when Hobbes formally asked the Royal Society's "judgment" about matters of mathematical foundations raised during the long, angry quarrel he and Wallis had been keeping up since 1656 ([19]). Rather unfairly the Society silently passed the questions to Wallis. He wrote the answers, which were anonymously published in the Philosophical Transactions. Hobbes asked six questions, three of which directly concerned the

\footnotetext{
${ }^{45}$ We cannot dwell here on the context of the squabble between Wallis and Hobbes. The bitterness in their mathematical disagreements derived from deep philosophical and political differences. On the wider conflict, cf. [46], passim. On the philosophical discussion about mathematical infinites, cf. [31], pp. 65-69, and [41].
} 
existence of infinites and infinitesimals ${ }^{46}$ :

2. Whether a Finite Quantity can be divided into an Infinite Number of lesser Quantities, or a Finite quantity consist of an Infinite Number of parts (which [Wallis] buildeth on as received from Cavallieri.)

3. Whether there be any Quantity greater than Infinite.

5. Whether there be any number Infinite. For it is one thing to say, that a Quantity may be divided perpetually without end, and another thing to say that a Quantity may be divided into an infinite number of parts.

Hobbes's questions, particularly number 5 , seem to point to the distinction between the actual and potential infinite division, that is to say, he seems to be asking whether Wallis stands for the actual infinite and the thesis that a quantity may be divided into an infinite number of parts. Wallis's answers rest on a dubious distinction between "to be" and "to be supposed to be" in mathematics. He claims that already in Euclid infinites are "supposed to be", although they are not taken to "actually be" (ibid.). His examples are straight lines and infinite division. Straight lines can be supposed as produced infinitely (by postulate I.2), even though this is not possible in practice ("it is not possible for any man to produce a straight line infinitely" (ibid.). Similarly, Euclid's proposition I.10, ensures that any segment may be bisected, and then each of the halves may as well, "and so onwards, Infinitely" (ibid.). This is not to be understood as a process to be actually done, but as a process that can be supposed as done, and therefore the infinite division can be supposed as well (ibid.):

Again, when (by Euclid's tenth Proposition) the same [straight line] AB, may be Bisected in $\mathrm{M}$ and each of the halves in $\mathrm{m}$, and so onwards, Infinitely: it is not his meaning [...] that it should be actually done, (for, who can do it?) but that it be supposed. And upon such (supposed) section infinitely continued, the parts must be (supposed) infinitely many.

Apparently Wallis is introducing a distinction between physical and logical impossibility. The existence of the actual infinite division of finite quantities (and therefore infinitesimals) is physically impossible, and yet is logically assumable. Wallis also claims Euclid to imply as much (ibid.) His answers to Hobbes's questions seem to be inspired by this, Wallis's version of the actual infinite (ibid., pp. 2242-3):

2. A Finite Quantity [...] may be supposed [...] divisible into a number of parts Infinitely many (or, more than any Finite number assignable:) [...]

3. Of supposed Infinites, one may be supposed greater than another: As a, supposed, infinite number of Men, may be supposed to have a Greater number of eyes [...]

5. There may be supposed a number Infinite; that is, greater than any assignable Finite: As the supposed number of parts, arising from a supposed Section Infinitely continued.

\footnotetext{
${ }^{46}$ We quote them separately, as printed in volume 6 of Philosophical Transactions (1671, Num. 75, September 18th, pp. 2241-2250, esp. p. 2242
} 
Wallis's notion of infinite, here, is consistent with the views on indivisibles and the method of exhaustion that he set forth a decade later in his Treatise of Algebra ([56]) and with his interpretation of the method of exhaustion. Having Hobbes's criticism in mind, in his Treatise of Algebra Wallis claimed that indivisibles were not points (in lines), or lines (in surfaces), or planes (in solids). Rather they had to be understood as infinitesimals ([56], pp. 285-286):

According to this Method [of lndivisibles], a Line is considered as consisting of an Innumerable Multitude of Points: A Surface, of Lines [...]: A Solid, of Plains, or other Surfaces [...]. Now this is not to be so understood, as if those Lines (which have no breadth) could fill up a Surface; or those Plains or Surfaces, (which have no thickness) could complete a Solid. But by such Lines are to be understood, small Surfaces, (of such a length, but very narrow,) [...].

Wallis clearly specified there that in this context 'very narrow' stands for 'infinitely narrow'.

In the same treatise ([56], p. 284) he analysed the foundations of the method of exhaustion, which, as Wallis puts it, shows that the difference between a given figure and other figures inscribed and circumscribed to it can be made "less than any assignable". Wallis stresses that this is not to be understood as if the given figure and the limit of the inscribed or circumscribed figures were equal more or less a negligible quantity. This, adds Wallis, is what Clavius understood, that in the limit the difference is so small as to become "heterogeneal" and not to meet "Archimedes's axiom" ([56], p. 284: here 'Archimedes's axiom' refers to proposition X.1 of Euclid's Elements). Wallis criticises generally Clavius's views on infinitely small elements, which Clavius understands as magnitudes heterogeneous with finite magnitudes. Wallis explicitly makes them homogeneous with finite magnitudes. Moreover, he envisions the series of inscribed and circumscribed figures that approximate a given figure as actually reaching it, in the sense that the final, limit figure is the last term of the series. In particular, this allows him to claim that the method of exhaustion in the limit yields full equality between the given figure and the limit of the approximating figures ([56], p. 284):

All continual approaches, in which the Distance comes to be less than any assignable, must be supposed, if infinitely continued, to determine in a Coincidence or Concurrence: [...]. Thus the Hyperbola and its Asymptote, if infinitely continued, must be supposed to meet [...]. Thus a Circle must be supposed Coincident with an (Inscribed or Circumscribed) Regular Polygone, of Sides infinitely many. And the like in cases Innumerable.

Wallis ended up his discussion of the "method of exhaustions" sending the reader to his Defense of the Treatise of the Angle of Contact, a short tract added to the Treatise of Algebra ([56], pp. 69-105 of the third page numbering; Latin Translation, with additions, in [57], vol. II, pp. 631-664), where he enlarges on his differences with Clavius.

\section{Discussing the Angle of Contingence}

Whether the angle of contingence (also called 'angle of contact' or 'horn angle') has to be considered an angle proper, and if yes how much it measures, had already been much debated when the matter gained notoriety through Peletier's and Clavius's discussion. 
Clavius published a rebuttal of Peletier's view in his influential commentary on Euclid's Elements ([37], pp. 73-77; [38] pp. 28-43; [8], ff. 110r -115v). Peletier answered this with a new short tract in 1579, and then Clavius came back once more against Peletiers views ([39]; [9], pp. 354-386.). Wallis criticised Clavius's views in 1656 in his De angulo contactus et semicirculi disquisitio geometrica ([52]). In 1663 this was in turn criticized by the Jesuit, Léotaud's Cyclomathia ([21], second separated pagination). Finally, Wallis answered to Léotaud with his just mentioned Defense of the Treatise of the Angle of Contact.

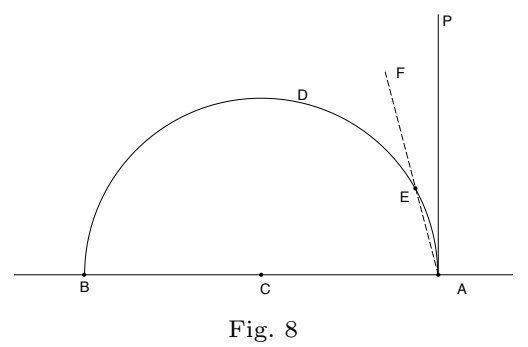

All the authors involved agreed, according to proposition III.16 of the Elements, that the horn angle $\widehat{\mathrm{DAP}}$ (fig. 8) is less than any acute rectilinear angle. This proposition actually states, also, that any straight line FA (different from the tangent PA) cuts the circumference of the circle at some point $E \neq A$. Now, according to Peletier, proposition III.16 is inconsistent with proposition X.l. The latter states that given two unequal magnitudes, by adding the smaller magnitude to itself a number of times it overcomes the larger one. (Literally, proposition X.l does not say so that, but Peletier, Clavius and Wallis took it to be equivalent to this result.) A learned humanist, a poet, and the author of several creative innovations regarding algebraic symbolism, Peletier wanted to remove that flaw from Euclid's text through a radical reinterpretation of the notions involved, namely by removing horn angles from the category of angle and by denying them the nature of quantities. Peletier stressed that otherwise Elements III.16 is in flagrant contradiction of Elements X.1 ([15], note to proposition III.16, vol. II, pp. 39-43; [22]). Notice that in Peletier's terms Euclid's Elements cannot be an undisputed reference - either it contains contradictions or its basic notions must be redefined.

Clavius's point is that angles of contingence must be assumed heterogeneous with rectilinear angles. However, as Peletier pointed out, Euclid explicitly compares the two kinds of angles, which makes them homogeneous magnitudes. To this, Clavius was to answer comparing horn angles to finite segments and rectilinear angles to infinite straight lines. Peletier countered Clavius's analogy by pointing out that infinite lines are no magnitudes the mathematician can handle. On the other hand, Clavius criticized Peletier's attempt at denying horn angles the status of true quantities by pointing to the obvious geometrical object made up by a curve and its tangent. They jointly determine a space endowed with well-defined geometrical properties. Consequently Clavius, although he did not clarify what kind of magnitudes they were, stood for his characterization of angles of contingency as magnitudes incomparably small compared with rectilinear angles ${ }^{47}$. Léotaud, following the lead of his teacher Tacquet, criticized his fellow

\footnotetext{
${ }^{47}$ Wallis fairly summarised Clavius's views in his Defense of the Treatise of the Angle of Contact: [56], pp. 74-79 of the third page numbering.
} 
Jesuit, Clavius, because Clavius let "incomparably small" quantities play a role in geometry ${ }^{48}$

Tacquet, one of the strongest and most consistent critics of the method of indivisibles, and then one of his disciples, Léotaud, criticized their fellow Jesuit, Clavius, because they wanted "incomparably small" quantities banned from geometry ([56], pp. 78-88 of the third page numbering).

Wallis's comments and criticism of Clavius's views illuminate his understanding of infinitely small parts. On the one hand, he claimed (in agreement with Clavius) that the angle of contingence was of "no magnitude" compared with any rectilinear angle. This was so because angles of contingence are always less than any rectilinear angle howsoever small ([56], p. 71 of the third page numbering; stress in the original):

In all sorts of Magnitudes (or Quantities) whatever, That which may be proved to be less than any assignable, is indeed (as to that sort of Quantity) of no Magnitude. (Because if of any, ... it might be so Multiplied as to exceed the greatest:).

On the other hand, he took pains to show that Clavius was wrong in making smallness the cause of the heterogeneity between angles of contingence and rectilinear angles. As we shall see, Wallis argued that Clavius's mistake originates in assuming that parts of a given (finite) magnitude can be heterogeneous with it because of too small; Wallis claimed that infinitely small parts keep their homogeneity.

Wallis agreed also with Clavius that angles of contingence are recognisable, well-determined geometrical objects. Therefore, Wallis's problem was how to allocate magnitude to angles of contingence to keep them heterogeneous with rectilinear angles.

Wallis brought into discussion the view - which he embraced elsewhere - that circular arcs are made up of infinitesimal chords. This view does him disservice here, since it might lead to understand the angle of contact as the addition of the infinitesimal angles between consecutive infinitesimal chords (or tangents). Therefore he dismisses it by means of a visual image in which the infinitesimal (rectilinear) angles eventually disappear when the infinitely sided polygon inscribed in the circle turns into the circle ([56], p. 91 of the third page numbering):

[Let a polygon be inscribed in a circle, and be] the number of sides infinitely many; such side must be infinitely short [...] and the External Angle infinitely small; but the Direction (or tendency) of such side (how small so ever) [...] must still be the same $[\ldots]$. But if then [...] such side (infinitely small) be supposed further to degenerate into a Point, and that Polygon into a Circle, [...] the Angle of Contact [...] which was, before, infinitely small, must now be nothing.

In order to introduce a magnitude for horn angles that is not homogeneous with the magnitudes of rectilinear angles, Wallis assumes a physicalist model patterned on the difference between speed and space. Wallis calls his basic notion 'inceptive of magnitude' and defines it as that thing which is nothing as to some magnitude and yet is the origin or beginning of it. His language, not straightforward, needs the examples that follow. First, his definition ([56], p. 95 of the third page numbering; stress in the original):

\footnotetext{
${ }^{48}$ Wallis summarised Léotaud's and Tacquet's views in [56], pp. $78-88$ of the third page numbering. On Tacquet, cf. [3] and [4].
} 
There are some things, which tho', as to some kind of Magnitude, they are nothing; yet are in the next possibility of being somewhat. They are not it, but tantum non; they are in the next possibility to it; and the Beginning of it: Tho' not as primum quod sit, (as the Schools speak) yet as ultimum quod non. And may very well be called Inchoactives or Inceptives, of that somewhat to which they are in such possibility.

A point in motion is inceptive of length (lines in motion are inceptives of surfaces, and so on). Yet in the same paragraph Wallis adds that speed ("Celerity or Swifteness") is also an inceptive of length, and acceleration is inceptive of "celerity". Wallis encapsulates the nature of rectilinear angles in "angular points", on the account that the angle is the same no matter how long its legs are. An "angular point" would show the degree of inclination between lines or the rate at which one line departs from another, which is the essence of a rectilinear angle. In that sense Wallis calls angles 'inceptives of distance' ([56], p. 97 of the third page numbering):

[Angle] is not distance: (like as, in motion, Celerity is not Length:) But it is Inceptive of distance; shewing the degree of Divarication, [or] Declination [...]: That is, at what rate $[\ldots]$ the line $A C$ doth divaricate, decline, deviate or depart from $A B$.

Rectilinear angles show the degree of "declination". When the "declination" is not constant we meet an angle of contingence, which gives the rate at which "declination" increases. (Wallis did not discuss mixtilineal angles in which the curve line crosses but is not tangent to the straight line.) Angles of contingence are no more rectilinear angles than speeds are distances, or accelerations speeds ([56], p. 98 of the third page numbering):

I say further, [...] That Deflection (whereby a Curve-line departs from its Tangent, and which is commonly called the Angle of Contact) is not Angle, or Declinatíon; (like as, in motion, Acceleration is not Celerity:) But is Inceptive of Declination; shewing the degree of Curvity: That is, at what rate... it flies off from Rectitude.

Generally speaking, says Wallis, inceptives have magnitudes of their own. Angles of contingence, in particular, have their own magnitudes enabling us to make comparisons between them (Wallis did not discuss the matter further; [56], pp. 96 and 99 of the third page numbering; the quote id from p. 99):

[Horn angles have their] Magnitude, tho of another kind and Heterogeneous to that of Angle; in like manner as Angle [...] is Heterogeneous to Distance; Celerity to Length; Acceleration to Celerity; Line to Surface.

Finally, having set forth his own original conceptualisation of angles of contingence, Wallis nicely summarises his points of agreement and disagreement with Clavius. They agree in that horn angles have a magnitude, and that it is heterogeneous to angle's. They disagree in that Clavius takes horn angles to be parts of rectilinear angles, and infinitely small ones ([56], p. 99 of the third page numbering):

I do thus far agree with Clavius, (and always did) That what he calls an Angle of Contact [...] hath a Quantity or Magnitude, capable of measure, [...]; and that [...] is Heterogeneous to Angle [...]; and therefore not capable of proportion to it, nor can by any Multiplication become equal to it, or exceed it. 
But herein we differ; That he makes his Angle of Contact, such a Quantity as is Part of a Rectilinear Right-angle; [...] and the Angle of Contact no otherwise Heterogeneous to a Right-lined Angle, but only because so very small.

And he concludes by spelling out that parts, even if infinitely small, are always homogeneous to the magnitude of which they are part ([56], p. 99 of the third page numbering; stress in the original):

Where, by the way, we may observe a great difference between the proportion of Infinite to Finite, and, of Finite to Nothing. For $\frac{1}{\infty}$, that which is a part infinitely small, may, by infinite Multiplication, equal the whole: But $\frac{0}{1}$, that which is Nothing, can by no Multiplication become equal to Something.

And this may serve for the settling of that Notion concerning the Angle of Contact, and other Notions of like Nature.

\section{Concluding Remarks}

It is impossible to be certain about Wallis's notion of indivisibles in the early years of his career, in the mid 1650s, when he wrote his fundamental De Sectionibus Conicis and Arithmetica infinitorum. His Mathesis universalis of the same years remains silent about indivisibles. When Wallis handled indivisibles in those early works, he almost always made room for both interpretationsindivisibles conceived either as heterogeneous elements or as infinitesimal homogeneous parts. The mathematical computations and arguments usually do not allow the reader to discriminate which interpretation was being used.

In later years, however, already in his discussions with Hobbes in the early 1670s and even more markedly in his discussion of the angle of contact in the first half of the 1680s, Wallis was embracing an infinitesimal understanding of indivisibles. It is impossible for us now to advance any conjecture about this shift, if indeed there was one, other than to point at the sharp differences between the mathematical contexts of the 1650s and 1680s. By the latter dates Leibniz had already published the first articles on his calculus, explicitly based on infinitesimals, and Newton's manuscripts (some of them) were in Wallis's hands. They might have influenced Wallis's views on indivisibles.

A final word on Wallis's vies on the angle of contingence is in order. 'Indivisibles' was an all-embracing, not too precise word that was applied to different things. However, in the second half of the 17th-century most people used the term to refer to infinitesimals rather than to Cavalierian indivisibles properly. Clavius did use indivisibles in conceptualising horn angles. He took them to be incomparably small to the whole to which they belonged according to the pattern of Cavalierian indivisibles, that is by assuming the indivisibles to be heterogeneous to the magnitudes comprising them (like a line and the surface in which the line lies are). Notice that Clavius's notion of incomparably small does not fit with later notions of mathematical smallness: according to Clavius, claiming that a geometrical entity is incomparably small than another is compatible with claiming (and even suggests) that these entities are heterogeneous to each other. While Wallis's solution to the conceptualisation of horn angles does not involve indivisibles or infinitesimals, yet his answer to Clavius's arguments contains important clarifications of his own views about these notions. He forcefully explained the crucial difference between being a 
part and being just "within" or "in" a magnitude. In doing so he was articulating a notion of mathematical smallness closer to ours. Perhaps more importantly, we find in his analysis of the angle of contact an intuition of the complexity of the relations between instantaneous variation and the incomparably small elements on which that variation depends. His notion of inceptives of magnitudes appears thereby grounded on his comprehensive exploration of the notions of indivisibles and infinitesimals.

\section{References}

[1] K. Andersen. Cavalieri's method of indivisibles. Archive for History of Exact Sciences, 31:291-367, 1985.

[2] M. E. Baron. The Origins of Infinitesimal Calculus. Pergamon Press, Oxford, 1969.

[3] H. Bosmans. Le jésuite math'ematicien anversois André Tacquet (1612-1660). Gulden Passer, 3:63-87, 1925.

[4] H. Bosmans. André Tacquet (S. J.) et son traité d'arithmétique théorique et practique. Isis, 8:66-82, 1927.

[5] F. Cajori. Controversies on mathematics between Wallis, Hobbes, and Barrow. Mathematics Teacher, 22:146-151, 1929.

[6] M. Cantor. Vorlesungen über Geschichte der Mathematik. B. G. Teubner, Leipzig, zweiten auflage edition, 1894-1901. 4 vols.

[7] B. Cavalieri. Exercitationes geometrica sex. Typis I. Montij, Bononiæ, 1647.

[8] C. Clavius. Euclidis Elementorum libri XV. apud V. Accoltum, Romæ, 1574.

[9] C. Clavius. Euclidis Elementorum libri XV. apud Bartholomaeum Grassium, Romæ, 1589.

[10] Grégoire de Saint Vincent. Opus geometricum quadraturce circuli et sectionis coni decem libris comprehensum. Apud Ioannem et Iacobvm Meursios, Antverpiæ, 1647.

[11] A. A. de Sarasa. Solutione problematis A. R. P. Marino Mersenno Minimo propositi [...]. Apud Ioannem et Iacobvm Meursios, Antverpiæ, 1649.

[12] J. Dhombres. L'innovation comme produit captif de la tradition: entre Apollonius et Descartes, une théorie des courbes chez Grégoire de Saint-Vincent. In M. Panza and C. S. Roero, editors, Geometria, flussioni e differenziali. Tradizione e innovazione nella matematica del Seicento, pages 13-83. La Città del Sole, Napoli, 1995.

[13] J. Dhombres. Les savoirs mathématiques et leurs pratiques culturelles. Tome 1: De l'âge baroque à la moisson des Lumières (1585-1750). Fayard, Paris, Forthcoming.

[14] A. N. F. Edwards. Pascal's Arithmetical Triangle. The Story of a Mathematical Idea. C. Griffin and Company, London, 1987. New edition (quoted): Johns Hopkins Univ. Press, Balimora, London, 2002. 
[15] Euclid. The Thirteen Books of the Elements. Cambridge Univ. Press, Cambridge, 2nd edition, 1926. Translated with introduction and commentary by Sir Thomas L. Heath; 3 vols.

[16] E. Giusti. Bonaventura Cavalieri and the Theory of Indivisibles. Cremonese, [Roma], 1980.

[17] N. Guicciardini. Isaac Newton on Mathematical Certainty and Method. Thde MIT Press, Cambridge (Mass), London, 2009.

[18] T. Hobbes. English Works of Thomas Hobbes. J. Bohn (vols. I-VI and VIII-IX), and Longman, Brown, Green, and Longmans (vols. VII and X-XI), London, 1839-1845. ed. by W. Molesworth. 11 vols.

[19] T. Hobbes. To the Right Honorable and others, the Learned Members of the Royal Society. n. p., n. d. [1671]. This is a folio leaflet printed on one side.

[20] D. M. Jesseph. Squaring the circle: the war between Hobbes and Wallis. Chicago Univ. Press, Chicago, 1999.

[21] V. Léotaud. Cyclomathia seu multiplex circuli contemplatio. sumptibus B. Cora, Lugduni, 1663.

[22] L. Maierù. “. . in Christophorum Clavium de Contactu Linearum Apologia”. Considerazioni attorno alla polemica fra Peletier e Clavio circa l'angolo di contatto (1579-1589). Archive for History of Exact Sciences, 41:115-137, 1990.

[23] L. Maierù. John Wallis. Una vita per un progetto. Rubbettino, Soveria Maqnnelli (CZ), 2007.

[24] A. Malet. From Indivisibles to Infinitesimals. Studies on Seventeenth-Century Mathematization of Infinitely Small Quantities. Servei de Publications de la Universitat Autònoma de Barcelona, Bellaterra, 1996.

[25] P. Mancosu. Philosophy of Mathematics and Mathematical Practice in the Seventeenth Century. Oxford Unversity Press, New York, Oxford, 1996.

[26] M. R. Massa. Mengoli on 'quasi proportions'. Historia Mathematica, 24:257-280, 1997.

[27] M. R. Massa. Algebra and geometry in Pietro Mengoli (1625-1686). Historia Mathematica, $33: 82-112,2006$.

[28] M. Mersenne. Cogitata physico mathematica [...]. Sumptibus Antonii Bertier, Parisiis, 1644.

[29] I. Newton. The Mathematical Papers of Isaac Newton. Cambridge University Press, Cambridge, 1967-1981. Edited by D. T. Whiteside (8 vols.).

[30] T. P. Nunn. The arithmetic of infinites. The Mathematical Gazette, 5:345-356 and 377-386, 1910-1911.

[31] E Vailati P. Mancosu. Torricelli's infinitely long solid and its philosophical reception in the seventeenth century. Isis, 82:50-70, 1991. 
[32] M. Panza. Da Wallis à Newton : una via verso il calcolo. quadrature, serie e rappresentazioni infinite delle quantità e delle forme trascendenti. In M. Panza and C. S. Roero, editors, Geometria, flussioni e differenziali. Tradizione e innovazione nella matematica del Seicento, pages 131-219. La Città del Sole, Napoli, 1995.

[33] M. Panza. Newton et les origines de l'analyse: 1664-1666. Blanchard, Paris, 2005.

[34] M. Panza. Nombres. Eléments de mathématiques pour philosophes. ENS edition, Lyon, 2007.

[35] M. Panza. Review of L. Maierù, John Wallis. Una vita per un progetto. Historia Mathematica, 36:279-281, 2009.

[36] B. Pascal. Traité du triangle arithmetique: avec quelques autres petits traitez sur la mesme matiere. G. Desprez, Paris, 1665.

[37] J. Peletier. In Euclidis elementa geometrica demonstrationum libri sex. apud Ioan. Tornæsium et Gul. Gazeium, Lugduni, 1557.

[38] J. Peletier. De mathematici commentarii libri tres. apud Joannem Oporinum, Basileæ, 1563.

[39] J. Peletier. In Christophorum Clavium De contactu linearum Apologia. apud H. de Marnef et Vm G. Cavellat, Parisiis, 1579.

[40] A. Prag. John Wallis. 1616-1703. zur Ideengeschichte der Matematik im 17. Jahrhundert. Quellen und Studien zur Geschichte der Mathematik, Astronomie und Physik, Abt. B, 1:381-412, 1931.

[41] S. Probst. Infinity and creation: the origin of the controversy between Thomas Hobbes and the Savilian professors Seth Ward and John Wallis. British Journal for the History of Science, 26:271-279, 1993.

[42] M. H. Pycior. Mathematics and Philosophy: Wallis, Hobbes, Barrow, and Berkeley. Journal of the History of Ideas, 48:265-286, 1987.

[43] S.J. Rigaud, editor. Correspondence of Scientific Men of Seventeenth Century. Oxford Univ. Press, Oxford, 1841. 2 vols.

[44] J. F. Scott. The Mathematical Work of John Wallis, D.D. F.R.S. (1616-1703). Taylor and Francis, LTD,, London, 1938.

[45] C. J. Scriba. Wallis, John. In Charles C. Gillispie, editor, Dictionary of Scientific Biography, volume XIV, pages 146-155. Charles Scribner's Sons, New York, 1976.

[46] S. Shapin and S. Schaffer. Leviathan and the air-pump. Princeton University Press, Princeton, 1985.

[47] J. A. Stedall. The discovery of wonders: Reading between the lines of John Wallis's Arithmetica Infinitorum. Archive for History of Exact Sciences, 56:1-28, 2001. 
[48] J. A. Stedall. A discourse Concerning Algebra : English Algebra to 1685. Oxford Univ. Press, Oxford, 2002.

[49] E. Torricelli. Opera geometrica Evangelista Torricellii [...]. Typis Amatoris Masse \& Laurentij de Landis, Florentiæ, 1644.

[50] J. Wallis. De Sectionibus Conicis Nova Methodo Expositis, Tractatus. typis L. Lichfield, Academiæ Typographi, Impensis T. Robinson, Oxonii, 1655. Included with separate pagination in [53]. Also in [57], vol. I, pp. 291-354.

[51] J. Wallis. Arithmetica infinitorum, Sive Nova Methodus Inquirendi in Curvilineorum Quadraturam, aliaque difficiliora Matheseos Problemata. typis L. Lichfield, Academiæ Typographi, Impensis T. Robinson, Oxonii, 1656. Included with separate pagination in [53]. Also in [57], vol. I, pp. 355-478.

[52] J. Wallis. De angulo contactus et semicirculi disquisitio geometrica. typis L. Lichfield, Academiæ Typographi, Impensis T. Robinson, Oxonii, 1656. Included with separate pagination in [53]. Also in [57], vol. II, pp. 603-630.

[53] J. Wallis. Operum Mathematicorum. Pars Altera [...]. typis L. Lichfield, Impensis T. Robinson, Oxonii, 1656.

[54] J. Wallis. Mathesis Universalis, Sive Arithmeticum Opus Inrtegrum [...]. typis L. Lichfield, Academiæ Typographi, Impensis T. Robinson, Oxonii, 1657. Included with separate pagination in [55]. Also in [57], vol. I, pp. 11-228.

[55] J. Wallis. Operum Mathematicorum. Pars Prima [...]. typis L. Lichfield, Impensis T. Robinson, Oxonii, 1657.

[56] J. Wallis. A Treatise of Algebra, both historical and practical [...]. With some additional Treatises. printed by John Playford, for Richard Davis, London, 1685. Latin Translation, with additions, in [57], vol. II, pp. [i]-482.

[57] J. Wallis. Opera Mathematica. E. Theatro Sheldoniano, Oxoniaæ, 1693-1699. 3 vols.

[58] J. Wallis. The Arithmetic of Infinitesimals. Springer, New York, etc., 2004. Translated from Latin to English with an Introduction by J. A. Stedall.

[59] D. T. Whiteside. Patterns of mathematical thought in the later seventeenth century. Archive for History of Exact Sciences, 1:179-388, 1960-1962. 